Claremont Colleges

Scholarship@ Claremont

All HMC Faculty Publications and Research

HMC Faculty Scholarship

8-1-1999

\title{
Black Holes and Five-brane Thermodynamics
}

Emil Martinec

University of Chicago

Vatche Sahakian

Harvey Mudd College

\section{Recommended Citation}

Emil Martinec and Vatche Sahakian. "Black holes and five-brane thermodynamics." Phys. Rev. D 60, 064002 (1999). doi: 10.1103/ PhysRevD.60.064002

This Article is brought to you for free and open access by the HMC Faculty Scholarship at Scholarship @ Claremont. It has been accepted for inclusion in All HMC Faculty Publications and Research by an authorized administrator of Scholarship @ Claremont. For more information, please contact scholarship@cuc.claremont.edu. 


\title{
Black holes and five-brane thermodynamics
}

\author{
Emil Martinec* and Vatche Sahakian ${ }^{\dagger}$ \\ Enrico Fermi Institute and Department of Physics, University of Chicago, 5640 S. Ellis Avenue, Chicago, Illinois 60637
}

(Received 24 February 1999; published 2 August 1999)

\begin{abstract}
The phase diagram for $\mathrm{D} p$-branes in $\mathrm{M}$ theory compactified on $\mathrm{T}^{4}, \mathrm{~T}^{4} / Z_{2}, \mathrm{~T}^{5}$, and $\mathrm{T}^{6}$ is constructed. As for the lower-dimensional tori considered in our previous work [E. Martinec and V. Sahakian, Phys. Rev. D 59, 124005 (1999)], the black brane phase at high entropy connects onto matrix theory at low entropy; we thus recover all known instances of matrix theory as consequences of the Maldacena conjecture. The difficulties that arise for $\mathrm{T}^{6}$ are reviewed. We also analyze the D1-D5 system on $\mathrm{T}^{5}$; we discuss its relation to matrix models of M5-branes, and use spectral flow as a tool to investigate the dependence of the phase structure on angular momentum. [S0556-2821(99)03316-0]

PACS number(s): 04.70.Dy
\end{abstract}

\section{SUMMARY OF RESULTS AND DISCUSSION}

\section{A. Introductory remarks}

Black hole thermodynamics has played an important role in elucidating the structure of $\mathrm{M}$ theory (see [1-3] for reviews). In the context of the Maldacena conjecture [4-6], black hole thermodynamics generates predictions for the thermodynamics of gauge theory in various strong-coupling regimes. This conjecture posits (in its extended form) that all of $\mathrm{M}$ theory in spacetimes with particular asymptotic boundary conditions is equivalent (dual) to a theory without gravity. Recently [7], the present authors constructed a phase diagram for maximally supersymmetric Yang-Mills (SYM) theory on tori $\mathrm{T}^{p}, p=1,2,3$, by systematically exploiting these ideas. It was seen that a number of different geometrical phases (i.e. those with a valid low-energy supergravity description as black objects) arise as the entropy and coupling are varied. ${ }^{1}$ The boundaries of the region of geometrical phases are correspondence curves [8], where the curvature of the geometry becomes string scale at the horizon of the object.

Generically, the thermodynamics at high entropy contains a phase of black D $p$-branes, while at low entropy one finds 11-dimensional black holes in the light-cone (LC) frame. The reason is quite simple [7]: The scaling limit specified by Maldacena and the limit prescribed by Sen and Seiberg for compactifications of matrix theory $[9,10]$ are one and the same. ${ }^{2}$ One and the same gauge theory describes both; for example, on $\mathrm{T}^{p}$, black $\mathrm{D} p$-branes characterize the density of states in the regime of SYM entropies $S \gtrsim N^{2}$, whereas matrix theory $[13,14]$ describes the regime $S<N$.

The point is that the scales of various features of the geometry, for instance proper size of the torus and the string

\footnotetext{
*Email address: ejm@theory.uchicago.edu

†Email address: isaak@theory.uchicago.edu

${ }^{1}$ The entropy is most useful in parametrizing the behavior of the theory since it is directly tied to the horizon area of the low-energy supergravity solution. The energy can then be read from the equation of state of the relevant black hole.

${ }^{2}$ It was shown that these two limits are related in [11,12]. Demonstrating their complete equivalence requires further specifying the dimensionless quantities to be held fixed, in particular the scale of the energy.
}

coupling, depend on the radial position in the associated lowenergy black supergravity solution. Since the horizon radius decreases with decreasing entropy, and only the horizon geometry is relevant to the thermodynamics, the entropy parametrizes a path through the moduli space of the low-energy supergravity. Along this path, it may be necessary to perform $U$-duality transformations to achieve a valid low-energy description of the horizon geometry. This is why, at high entropy, the charge carried by the system is the $\mathrm{D} p$-brane number, while at low entropy, it is interpreted as momentum. The two lie on an orbit of the $U$-duality group $E_{p}(\mathbf{Z})$. Furthermore, phase transitions may occur in the geometrical region due to (de)localization of the horizon on cycles across which it is initially (un)smeared [15]. Such transitions are involved in the passage from black $\mathrm{D} p$-branes to matrix theory black holes [16-19].

Thermodynamics is one of many probes of matrixMaldacena duality. It is a particularly useful one in that it canonically associates an energy scale (that of a typical Hawking quantum) with a particular place in the geometry (the horizon). The fact that the geometry appropriate to the description of this scale undergoes a sequence of duality transformations as we go from IR (matrix theory regime) to UV (Maldacena regime) means that the interpretation of probes as scattering states in discrete LC quantization (DLCQ) M theory is only valid up to some scale, beyond which one should pass to a description in terms of scattering off of black $p$-branes in a dual geometry. Using the relation between the energy and the radial scale probed [20], this implies that matrix theory is only valid (in the sense of accurately describing flat-space supergravity) up to some distance from the source. ${ }^{3}$

The precise relation between the Maldacena or nearhorizon limit of $N \mathrm{D} p$-branes on $\mathrm{T}^{p}$ and matrix theory on $\mathrm{T}^{p}$

\footnotetext{
${ }^{3}$ This conclusion was independently reached from a somewhat different perspective in [21]. The analysis of supersymmetric quantum mechanics (SQM) in this latter work is equivalent to the large $V$ limit of the phase diagrams here and in [7]. In Sec. II D of [7] it was observed that the D0 geometry breaks down at the correspondence point, where the temperature of the system is $T$ $\sim N^{1 / 3} R_{11} 1 / l_{\mathrm{pl}}^{2}$. Using the energy-distance relations of [20], the result $r_{\max } \sim N^{\mathrm{l} / 3} l_{\mathrm{pl}}$ follows.
} 
with $N$ units of longitudinal momentum goes as follows [7]: The Maldacena limit is $\alpha^{\prime}=l_{s t r}^{2} \rightarrow 0$, with the gauge coupling $g_{Y}^{2}=g_{s t r} l_{s t r}^{(p-3) / 2}$ and the coordinate size $\Sigma_{i}$ of the torus cycles held fixed. This limit isolates the gauge theory dynamics on the $\mathrm{D} p$-brane while decoupling gravity (for $p$ $<6$ ). Natural energy scales in the gauge theory are measured with respect to the torus size. ${ }^{4}$ On the other hand, the Seiberg-Sen prescription for matrix theory on $\mathrm{T}^{p}[9,10]$ involves type IIA string theory with $N$ D0-branes or, equivalently, M theory with $N$ units of momentum on a circle of radius $R_{11}$; then one takes the limit $l_{\mathrm{pl}} \rightarrow 0$, with $R_{11} / l_{\mathrm{pl}}^{2}$ and the (transverse) torus cycle sizes $R_{i} / l_{\mathrm{pl}}$ held fixed. The relation between the two sets of parameters is simply (cf. $[11,12,7])$ the $T$ duality on all cycles of $\mathrm{T}^{p}$ that maps $\mathrm{D} p$-branes to D0-branes and vice versa:

$$
\begin{aligned}
l_{s t r}^{2} & =\frac{l_{\mathrm{pl}}^{3}}{R_{11}} \\
\Sigma_{i} & =\frac{l_{\mathrm{pl}}^{3}}{R_{11} R_{i}} \\
g_{s t r} & =\left(\frac{l_{\mathrm{pl}}}{R_{11}}\right)^{(p-3) / 2} \prod_{i=1}^{p} \frac{l_{\mathrm{pl}}}{R_{i}} \\
g_{Y}^{2} & =\left(\frac{l_{\mathrm{pl}}^{2}}{R_{11}}\right)^{p-3} \prod_{i=1}^{p} \frac{l_{\mathrm{pl}}}{R_{i}} .
\end{aligned}
$$

Thus, the two limits are clearly identical.

In this work, we extend our analysis of such compactifications to $p=4,5$, where the relevant theories involve the dynamics of five-branes $[22,23,9,10]$, and $p=6$, where the definition of matrix theory is problematic $[9,10,24-26]$. In the process of generating the phase diagram, we will rediscover all the remaining prescriptions for generating matrix theory compactifications; we will also comment on the difficulties encountered for $p=6$ (and a proposal by Kachru et al. [27] for overcoming them). For $p=5$, we map out the phase diagram of the six-dimensional "little string theories" compactified on a five-torus $\mathrm{T}^{5}$.

In addition, we will analyze the phase diagram of the D1-D5 system, which arises in diverse contexts:

It has played a central role in our understanding of black hole thermodynamics [28].

It is a prime example of the Maldacena conjecture, due to the rich algebraic structure of $(1+1) \mathrm{D}$ superconformal theories which are proposed duals to string theory on $\mathrm{AdS}_{3} \times \mathrm{S}^{3} \times \mathcal{M}_{4}[4,29-31]$.

It describes the "little string" theory of five-branes $[23,32]$, where the little strings carry both winding and momentum charges.

\footnotetext{
${ }^{4}$ For $p \neq 3$, the Yang-Mills coupling is dimensionful, and should be referred to the torus scale as well. When we say that a dimensionful quantity is held fixed in the decoupling limit, we mean the energy in the system relative to that scale.
}

It is related to the DLCQ description of five-brane dynamics $[33-36]$.

The analysis will clarify the relation of the D-brane description of the system to one in terms of NS five-branes and fundamental strings [31], as low-energy descriptions of different regions of the phase diagram (for earlier work, see [37]). Finally, we will explore the use of spectral flow in the superconformal theory to determine the spectral density of the theory as a function of angular momentum on $\mathrm{S}^{3}$.

\section{B. Phase diagrams for $T^{4}, T^{5}$, and $T^{6}$}

As in [7], the phase diagrams for $\mathrm{D} p$-branes on tori, $p$ $=4,5,6$, have a number of common features. The vertical axis of the diagrams will be entropy; for the horizontal axis we take the size $V$ of cycles on the torus $\mathrm{T}^{p}$ in 11dimensional Planck units, as measured in the LC M theory appearing in the lower right corner (the phase of boosted 11D black holes). $N$ is the charge carried by the system: the brane number in the high entropy regimes and longitudinal momentum in the low-energy, LC M-theory phase. Throughout the various phases, the corresponding gravitational couplings vanish in the Maldacena limit (except for $p=6$, where the limit keeps the Planck scale of the high-entropy phase held fixed), implying the decoupling of gravity for the dual dynamics. Solid lines in the diagrams denote thermodynamic transitions separating distinct phases, while dotted lines represent symmetry transformations which change the appropriate low-energy description. We do not expect sharp phase transitions along these dotted curves since the scaling of the equations of state is unchanged across them. ${ }^{5}$

The structure of the phase diagram for $V>1$ is identical to the cases encountered in [7] (see, for example, Fig. 1). At high entropies and large M-theory $\mathrm{T}^{p}$, we have a perturbative $(p+1) \mathrm{D}$ SYM gas phase. Its Yang-Mills coupling $g_{Y}$ increases toward the left, cf. Eq. (1). The effective dimensionless coupling is of order 1 on the double lines bounding this phase, which are Horowitz-Polchinski correspondence curves. As the entropy decreases at large $V$, there is a D0brane phase arising on the right and middle of the diagrams. Its description as a thermodynamic state within SYM theory would be highly interesting. It has a Horowitz-Polchinski correspondence point at $S \sim N^{2}$, where a zero specific heat transition is to occur [38], and localizes into a LC 11D black hole phase for entropies $S<N$. The line $S \sim N$ separates the 11D phases that are localized on the M-theory circle (whose coordinate size is $R_{11}$ ) from those that are delocalized, uniformly across the diagram [16-19]. The 11D black hole phase at small entropy becomes smeared across the $\mathrm{T}^{p}$ when the horizon size becomes smaller than the torus scale $V$; we denote generally such smeared phases by an overline (in this case $\overline{11 d}$ ). This (de)localization transition of the horizon on the compact space extends above the $S \sim N$ transition, separating the black $\mathrm{D} p$-brane phase from the black D0-brane

\footnotetext{
${ }^{5}$ This does not in principle exclude the possibility of smoother (i.e. higher order) transitions.
} 
phase. ${ }^{6}$ Susskind [39] has argued that, on the SYM side, one should regard this localization transition as an analogue of the Gross-Witten large $N$ transition [40]. The localization transition line runs into the correspondence curve separating the SYM gas phase from the geometrical phases at $S \sim N^{2}$. Thus as we move to the left (decreasing $V$, i.e. increasing the bare SYM coupling) at high entropy $S>N^{2}$, the SYM gas phase reaches a correspondence point; on the other side of the transition is the phase of black $\mathrm{D} p$-branes. A further common feature of the diagrams is a "self-duality" point at $V \sim 1$ and $S \sim N^{(8-p) /(7-p)}$, where a number of $U$-duality curves meet.

In contrast, the structure of the phase diagrams for $V<1$ depends very much on the specific case at hand. Compactifications on $\mathrm{T}^{p}, p=1,2,3$, were analyzed in [7]; we now describe the specifics of this region for $p=4,5,6$.

Figure 1 is the phase diagram of $\mathrm{T}^{4}$ compactification. There are six different phases, several of which-the 11D and $\overline{11 \mathrm{~d}}$ black hole, black D0- and D $p$-branes, and SYM gas phases-were discussed above. In a slight shift of emphasis, we have relabeled the black D4-brane phase as the black M5-brane phase, since its description in terms of the latter object extends to the region $V<1$ (in fact, even for a patch of $V>1$ the D4-brane becomes strongly coupled and must be lifted to $\mathrm{M}$ theory). The appropriate dual non-gravitational description involves the six-dimensional $(2,0)$ field theory on $\mathrm{T}^{4} \times \mathrm{S}^{1}$, where the last factor is the M-theory circle; the scale of Kaluza-Klein excitations given by the size of this circle (times the number of branes) sets the transition point between the $(2,0)$ and SYM descriptions. This M5 phase consists of six patches that we cycle through via duality transformations required to maintain a valid low-energy description. The energy per entropy increases toward the left and toward higher entropies; this is to be contrasted with the cases analyzed in [7] where the IR limit appears toward the left of the diagrams. This behavior is a consequence of the reversal of the direction of renormalization group (RG) flow between $p<3$ and $p>3$. As we continue to the left and/or down on the figure at small volume $V<1$, the $\mathrm{T}^{4}$ is small while the M-theory circle remains large; eventually one reduces string theory along the cycles of the $\mathrm{T}^{4}$, and the M5brane dualizes into a string. Somewhat further in this direction, we encounter a Horowitz-Polchinski correspondence curve, and a transition to a phase consisting of a matrix string [41-43] with the effective string tension set by the adjacent geometries. Using Maldacena's conjecture, we thus validate earlier suggestions to describe matrix strings using the $(2,0)$ theory $[22,23,9]$. This matrix string phase has a correspondence curve also for low entropies, now with respect to a phase of smeared LC M-theory black holes (or

\footnotetext{
${ }^{6}$ Initially, the D0-brane phase becomes smeared to $\overline{\mathrm{D} 0}$; as the entropy increases, the effective geometry of the latter patch becomes substringy at the horizon, and one should $T$ dualize into the black $\mathrm{D} p$-brane patch. Both the $\overline{\mathrm{D} 0}$ and $\mathrm{D} p$ patches have the same equation of state, since they are related by a symmetry transformation of the theory; they are different patches of the same phase.
}

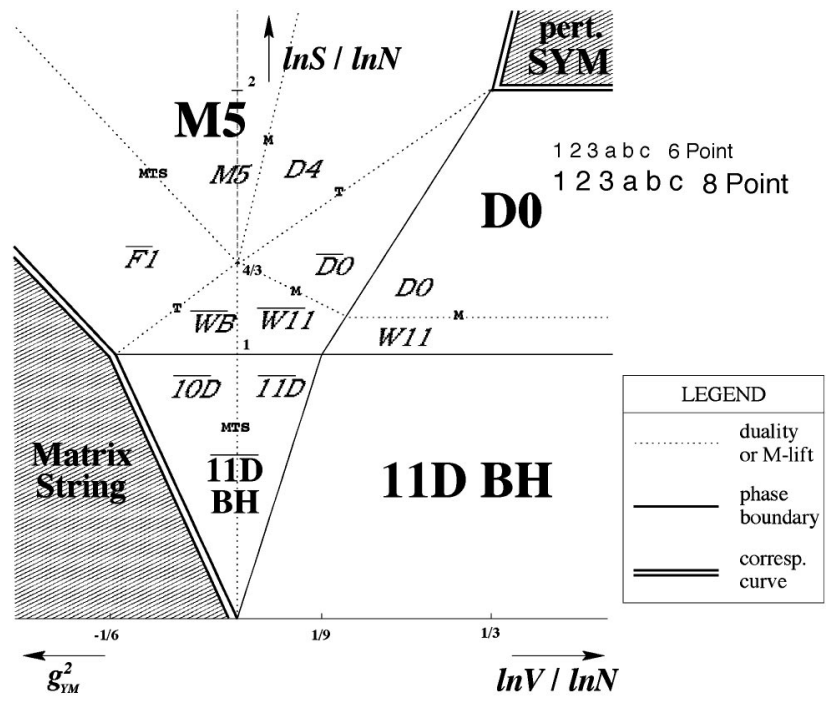

FIG. 1. Phase diagram of the six-dimensional $(2,0)$ theory on $\mathrm{T}^{4} \times \mathrm{S}^{1} . S$ is entropy, $V=R / l_{\mathrm{pl}}$ is the size of a cycle on the $\mathrm{T}^{4}$ of light-cone $\mathrm{M}$ theory, and $N$ is longitudinal momentum quantum. The dotted lines denote symmetry transformations: M, M lift or reduction; $T, T$ duality; $S, S$ duality. The solid lines are phase transition curves. Double solid lines denote correspondence curves. The dashed line is the extension of the axis $V=1$, and is merely included to help guide the eye. The labels are defined as follows: D0, black D0 geometry; W11, black 11D wave geometry; 11DBH, 11D LC black hole; $\overline{\mathrm{D} 0}$, black smeared D0 geometry; $\overline{\mathrm{W} 11}$, black smeared 11D wave geometry; $11 \mathrm{DBH}, 11 \mathrm{D}$ smeared LC black hole; D4, black D4 geometry; M5, black M5 geometry; $\overline{\mathrm{F} 1}$, black smeared fundamental string geometry; $\overline{\mathrm{WB}}$, black smeared type IIB wave geometry; $10 \mathrm{DBH}$, type IIB boosted black hole. The phase diagram can also be considered that of the $(2,0)$ theory on $\mathrm{T}^{4} / Z_{2}$ $\times \mathrm{S}^{1}$ by reinterpreting the $\overline{\mathrm{F}} 1, \overline{\mathrm{WB}}, \overline{10 \mathrm{D}}$ phases, and the matrix string phase as those of a Heterotic theory.

equivalently boosted type IIB holes).

Figure 1 is trivially modified to give the phase diagram of the $(2,0)$ theory on $\mathrm{T}^{4} / Z_{2} \times \mathrm{S}^{1}$. The additional structure does not affect the critical behavior of the diagram. The change appears in the chain of dualities we perform on the dotted lines of the diagram. The orbifold quotient metamorphoses into world-sheet parity, and the fundamental string patch (labeled $\overline{\mathrm{F} 1}$ ) becomes that of the heterotic string. The emerging matrix string phase at the correspondence point is then that of a heterotic theory. We thus confirm the suggestion $[44,45]$ to describe heterotic matrix strings via the $(2,0)$ theory on $\mathrm{T}^{4} / Z_{2} \times \mathrm{S}^{1}$. One can also propose to extend the dual theory of an intermediate state obtained in the chain of dualities between the M5 and the $\overline{\mathrm{F} 1}$ patches into the matrix string regime; we then have heterotic matrix strings encoded in the $O(N)$ theory of type-I D-strings, as suggested in [46-48]. Similar statements can be made about matrix theory orbifolds or orientifolds in other dimensions.

The thermodynamic phase diagram of five-branes (sometimes called the theory of "little strings" [49,23,32]) on $\mathrm{T}^{5}$ is shown in Fig. 2. We have a total of seven distinct phases. We again shift the notation somewhat, relabeling the black 


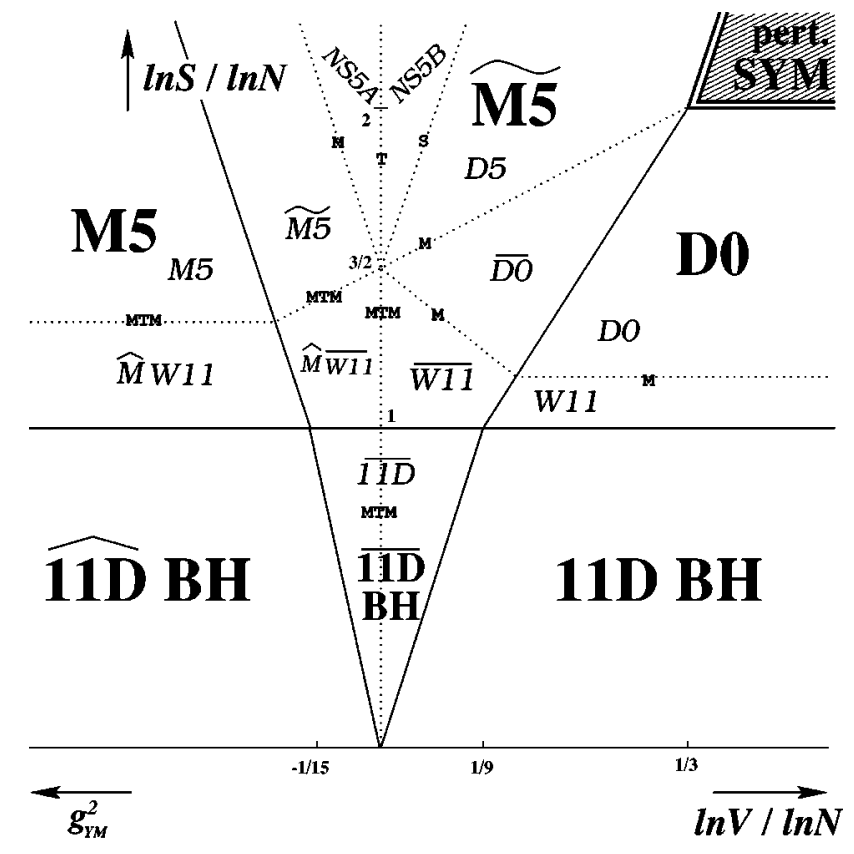

FIG. 2. Phase diagram of "little string" theory on $\mathrm{T}^{5}$. The labeling is as in Fig. 1. D0, black D0 geometry; W11, black 11D wave geometry; 11DBH, 11D LC black hole; $\overline{\mathrm{D} 0}$, black smeared D0 geometry; $\overline{\mathrm{W} 11}$, black smeared $11 \mathrm{D}$ wave geometry; $\overline{11 \mathrm{D} B \mathrm{H}}$, 11D smeared LC black hole; D5, black D5 geometry; NS5B, black five branes in type IIB theory; NS5A, black five-branes in type IIA

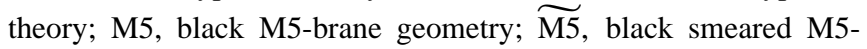
brane geometry; $\hat{\mathrm{M}} \overline{\mathrm{W} 11}$, black smeared wave geometry in $\hat{\mathrm{M}}$ theory; $\hat{\mathrm{M} W 11}$, black smeared wave geometry in $\hat{\mathrm{M}}$ theory; $\widehat{11 D B H}$, smeared boosted black holes in $\hat{\mathrm{M}}$ theory.

D5 phase as a black $\widetilde{\mathrm{M} 5}$ phase, since the latter extends the validity of the description to ${ }^{7} V<1$. The equation of state of this high-entropy regime is

$$
S \sim E N^{1 / 2}\left(\frac{l_{\mathrm{pl}}^{2}}{R_{11}}\right) V^{-5 / 2},
$$

characteristic of a string in its Hagedorn phase. The temperature determines the tension of the effective string. We have a patch of black NS5-branes in the middle of the diagram. They appear near the $V \sim 1$ line, at which point a $T$-duality transformation exchanges five-branes in type IIA and IIB theories. The type IIB Neveu-Schwarz five-brane (NS5) patch connects to a D5-brane patch via $S$ duality. The type IIA NS5 patch lifts to a patch of M5-branes on $\mathrm{T}^{5} \times \mathrm{S}^{1}$ at strong coupling on the left. The extra circle is the $\mathrm{M}$ circle transverse to the wrapped M5-branes; the horizon undergoes a localization transition on this circle at lower entropy and/or

\footnotetext{
${ }^{7}$ The tilde is meant to distinguish this 11-dimensional phase (where the $\mathrm{M}$ circle is transverse to the five-branes) from the 11dimensional LC phase on the lower right, whose $\mathrm{M}$ circle has a different origin.
}

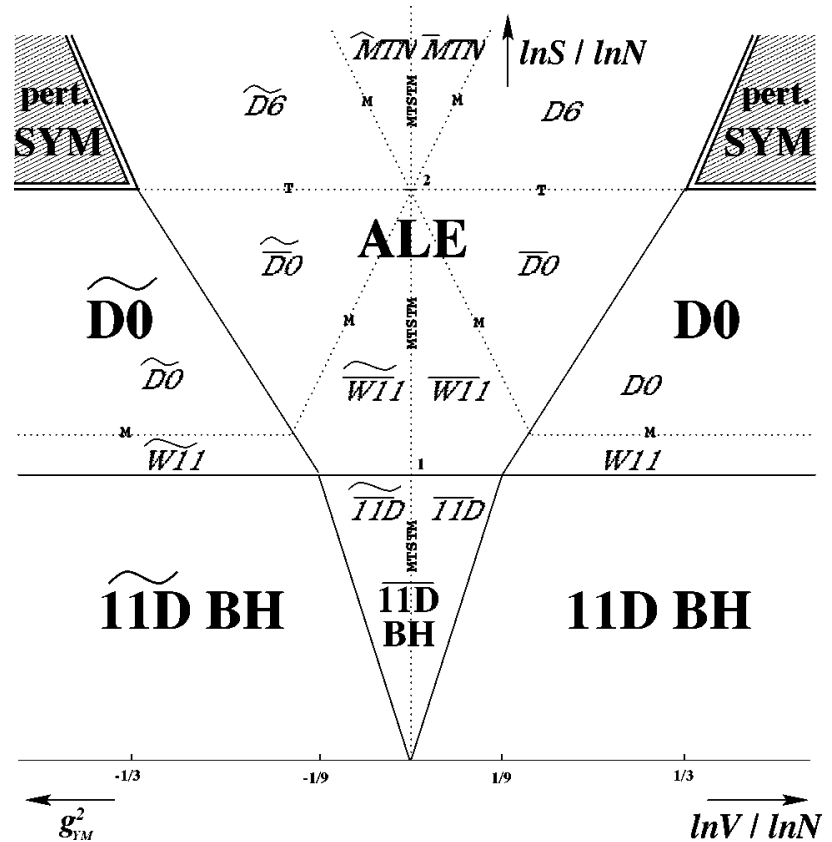

FIG. 3. Phase diagram of the D6 system. $S$ is entropy, $V$ $=R / l_{\mathrm{pl}}$ is the size of a cycle on the $\mathrm{T}^{6}$ of the LC M theory, and $N$ is longitudinal momentum. The dotted lines are symmetry transformations: M, M lift or reduction; $T, T$ duality; $S, S$ duality. The solid lines are phase transition curves. Double solid lines denote correspondence curves. The labels are defined as follows: $\bar{M} T N$, MTN, black Taub-NUT geometry; D6, $\widetilde{D 6}$, black D6 geometry; D0, $\widetilde{\mathrm{D} 0}$, black D0 geometry; W11, $\overparen{\mathrm{W} 11}$, black 11D wave geometry; 11DBH, $11 \mathrm{DBH}, 11 \mathrm{D}$ LC black hole; $\overline{\mathrm{D} 0}, \overline{\mathrm{D} 0}$, black smeared D0 geometry; $\overline{\mathrm{W} 11}, \overline{\mathrm{W} 11}$, black smeared 11D wave geometry; 11DBH, 11DBH, 11D smeared LC black hole.

smaller $V$ to a phase whose equation of state is that of a ( 5 $+1) \mathrm{D}$ gas. It is interesting that the Hagedorn transition is seen here as a localization-delocalization transition in the black geometry. Yet further in this direction, the system localizes at $N \sim S$ to a dual LC $\hat{\mathrm{M}}$ theory on a $\mathrm{T}^{4} \times \mathrm{S}^{1} \times \mathrm{S}^{1}$; here the horizon is smeared along the square $\mathrm{T}^{4}$, localized along both $\mathrm{S}^{1}$ factors, and carrying momentum along the last $\mathrm{S}^{1}$. This $\hat{M}$ phase on the lower left is $U$ dual to the LC M theory on the lower right.

The D6 phase diagram has two important features (see Fig. 3). First of all, the Maldacena limit keeps fixed the Planck scale $\tilde{l}_{\mathrm{pl}} \sim\left(l_{\mathrm{pl}}^{2} / R_{11}\right) V^{-2}$ of the high-entropy black Taub-Newman-Unti-Tamburino (Taub-NUT) geometry ${ }^{8}$ [50]. Thus, gravity does not decouple, and the limit does not lead to a non-gravitational dual system that would serve as the definition of $\mathrm{M}$ theory in such a spacetime. A symptom of this lack of decoupling of gravity is the negative specific heat $S \propto E^{3 / 2}$ of the high-entropy equation of state. This property is related to the breakdown of the usual UV-IR corre-

\footnotetext{
${ }^{8}$ In the Maldacena limit, the near horizon geometry is that of an asymptotically locally Euclidean (ALE) space with $A_{N-1}$ singularity.
} 
spondence of Maldacena duality [51,20]. The energy-radius relation of [20] determined by an analysis of the scalar wave equation in the relevant supergravity background is in fact the relation between the horizon radius and the Hawking temperature of the associated black geometry; thus, for $p$ $=6$ decreasing energy of the Hawking quanta is correlated to increasing radius of the horizon, as a consequence of the negative specific heat. This is to be contrasted with the situation for $p<5$, where the positive specific heat means increasing horizon radius correlates to increasing temperature, and $p=5$, where the Hawking temperature is independent of the horizon radius in the high-entropy regime. Now, the temperature in any dual description must be the same as in the supergravity description. For $p<5$, the dual is a field theory; high temperature means UV physics dominates the typical interactions, leading to the UV-IR correspondence. For $p$ $=5$, the dual is a "little string" theory; the temperature is unrelated to the horizon radius (and thus the total energy) on the gravity side, and unrelated to short-distance physics in the dual "little string" theory (since high-energy collisions of strings do not probe short distances). Hence the UV-IR correspondence already breaks down at this point. For $p$ $=6$, there is nothing to say-large radius (large total energy) corresponds to low temperature of probes (Hawking quanta), and any dual description could not have high energy or temperature related to short distance physics, since it is a theory that contains gravity (so high energy makes big black holes).

A second key feature is the duality symmetry (cf. [52]) $V \rightarrow V^{-1}$ of the diagram relating the $V<1$ structure to that discussed above for $V>1$. Note that this duality symmetry inverts the $\mathrm{T}^{6}$ volume as measured in Planck units rather than string units. The duality interchanges momentum modes with five-brane wrapping modes, while leaving membrane wrapping modes fixed; in other words, the dual space is that seen by the M5-brane. It is possible that this symmetry extends to any Calabi-Yau compactification of M theory, since the volume of the Calabi-Yau sits in a universal hypermultiplet whose moduli space appears to be $S U(2,1) / U(2)$ [53]; if the discrete identifications involve the appropriate element of $S U(2,1 ; \mathbf{Z})$, there will be a dual Calabi-Yau compactification of roughly the inverse size seen by M-theory fivebranes wrapping the original Calabi-Yau compactification.

The thermodynamic perspective also sheds light on a proposal of Kachru, Lawrence, and Silverstein [27] for a definition of matrix theory compactifications on Calabi-Yau spaces. Generically, string theory on a Calabi-Yau space does not have a $T$ duality that inverts its volume in string units. Rather, these authors suggest that the appropriate duality to consider, analogous to the $T$-duality transformation used by Sen-Seiberg for torus compactifications, is the mirror symmetry transformation. This transformation relates D0-branes in type IIA theory on a given Calabi-Yau space to D3-branes wrapping a special Lagrangian submanifold of the type IIB mirror [54]; locally, the Calabi-Yau space looks like a $\mathrm{T}^{3}$ fibered over an $\mathrm{S}^{3}$ base, and mirror symmetry is $T$ duality on the fiber. Thus, it is proposed that some sort of $3+1$ gauge dynamics might yield an appropriate underlying description. Consider the phase diagram that should arise. At low entropy, one has the 11D black hole phase. As the en- tropy increases at fixed but not large Calabi-Yau coordinate size $V$, one finds the horizon smears over the Calabi-Yau space and eventually one reaches the $\overline{\mathrm{D} 0}$ patch of smeared black D0-branes. The proper size of the Calabi-Yau space at the horizon in string units is decreasing along this path; eventually one reaches the curve along which one should perform the duality transformation, in this case mirror symmetry. Naively, in the mirror, as the entropy increases further, the $\mathrm{T}^{3}$ wrapped by the D3-branes is increasing in size, while the base $\mathrm{S}^{3}$ continues to shrink; the high-entropy phase would seem to be described by D3-branes on the special Lagrangian cycle of the mirror Calabi-Yau space near a conifold singularity. However, the duality transformation will not change the equation of state, since the $\overline{\mathrm{D} 0}$ patch and everything above it are related by symmetries of the theory. The only thing that could change this conclusion is a further phase transition, but there is no candidate. We conclude that the high-entropy phase is again one with negative specific heat, and thus cannot be that of a field theory.

\section{D1-D5 system}

As a further example of our methods, we have examined the D1-D5 system on $\mathrm{T}^{4} \times \mathrm{S}^{1}$, which as we mentioned above can be considered as the "little string" theory of $Q_{5}$ fivebranes, with $Q_{1}$ units of string winding along the $\mathrm{S}^{1}$. Figure 4 shows the thermodynamic phase diagram. In the Maldacena limit, this theory is a representation of the algebra of $\mathcal{N}=(4,4)$ superconformal transformations in $(1+1) \mathrm{D}$ $[4,55,56,29-31]$. We have defined $k \equiv Q_{1} Q_{5}$ and $q$ $\equiv Q_{1} / Q_{5}$. We keep $k$ fixed, but $q$ may be viewed as a variable in the range $1<q<k$, thus moving some of the dotted curves of duality transformation, but not altering phase transition curves. For $q \sim 1$, we can exchange the roles of $Q_{1}$ and $Q_{5}$ via duality transformations across the diagram; the structure is unchanged. The other limit, $q=k$, is the $Q_{5}=1$ bound. The vertical axis on the diagram is again the entropy, while the horizontal axis is the six-dimensional string coupling $g_{6} \equiv g_{s} / \sqrt{\mathbf{v}}$ of the D1-D5 patch, where $\mathbf{v}=V_{4} / \alpha^{\prime 2}$ is the volume of the $\mathrm{T}^{4}$ in string units [equivalently $g_{6}^{-2}$ is the volume of the $\mathrm{T}^{4}$ in appropriate string units of the NS5 fivebrane (NS5FB) phase]. The phase diagram has a symmetry $g_{6} \rightarrow 1 / g_{6}$ (inversion of the torus in the NS5FB phase); this is the $T$-duality symmetry of the little string theory. From the perspective of the D1-D5 patch, we can consider the entire phase diagram as that of the $1+1 \mathrm{D}$ conformal theory that arises in the IR of this gauge theory, which is conjectured to be dual to the near-horizon geometry $\operatorname{AdS}_{3} \times \mathrm{S}^{3} \times \mathrm{T}^{4}$ of the D1-D5 system. In this patch, the D-strings are wrapped on a cycle of size $R_{5}$. This parameter is absent from the scaling relations of all curves because of conformal symmetry.

\footnotetext{
${ }^{9}$ Note that one could also imagine performing the same duality sequence to describe matrix theory on K3 in terms of two-branes on the torus fiber of a near-degenerate mirror K3. In this case one knows that this description is related to the five-brane description given above by duality, and hence indeed has a $(5+1) \mathrm{D}$ equation of state at high entropy, rather than $\mathrm{a}(2+1) \mathrm{D}$ equation of state.
} 


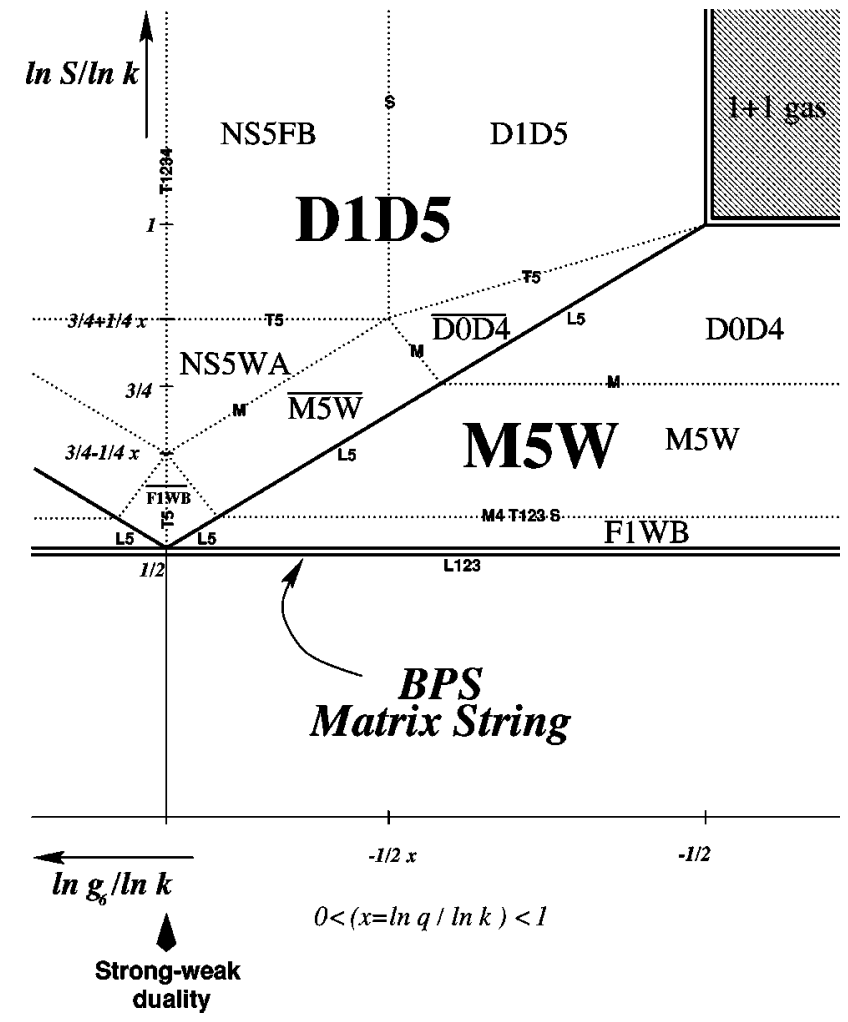

FIG. 4. Thermodynamic phase diagram of "little strings" wound on the $\mathrm{S}^{1}$ of $\mathrm{T}^{4} \times \mathrm{S}^{1}$, with $Q_{1}$ units of winding and $Q_{5}$ five-branes. $k \equiv Q_{1} Q_{5}$ and $1<q \equiv Q_{1} / Q_{5}<k . g_{6}$ is the six dimensional string coupling of the D1-D5 phase. The labels are defined as follows: D1D5, black D1-D5 geometry; NS5FB, black NS5 geometry with fundamental strings in type IIB theory; D0D4, black D0-D4 geometry; $\overline{\mathrm{D} 0 \mathrm{D} 4}$, black smeared D0-D4 geometry; M5W, black boosted M5-brane geometry; $\overline{\mathrm{M} 5 \mathrm{~W}}$, black smeared boosted M5-brane geometry; NS5WA, black boosted NS5 branes in type IIA theory; F1WB, black boosted fundamental strings in type IIB theory; $\overline{\mathrm{F} 1 \mathrm{WB}}$, black smeared and boosted fundamental strings in type IIB theory; $L$, localization transitions.

Analogous to the singly charged brane systems we have been discussing, at high entropies there is a " $(1+1) \mathrm{D}$ gas" phase at small $g_{6}\left(\right.$ large $\left.V_{4}\right)$, which passes across a correspondence curve to the black brane phase as the coupling increases. Being determined by conformal symmetry and quantization of the central charge, the equation of state does not change across this "phase transition." Starting in the " $(1+1) \mathrm{D}$ gas" phase and decreasing the entropy, $S \sim k$ corresponds to the point where the thermal wavelength in the $(1+1) \mathrm{D}$ conformal theory becomes of the order of the size of the box $R_{5}$. This is again a Horowitz-Polchinski correspondence curve from the side of lower entropies, analogous to the SYM theories at ${ }^{10} S \sim N^{2}$. There is a localization transition on the $R_{5}$ cycle cutting obliquely across the diagram. The localized phase can be interpreted as that of M5-branes with a large

\footnotetext{
${ }^{10}$ There is similarly a hidden phases of zero specific heat between the gas phase and the lower, localized phase, as can be seen by the discontinuity in temperatures that occurs between $S>k$ and $S<k$.
}

boost, thus connecting with the proposal of [33] for a matrix theory of this system. The lower boundary of this phase occurs at entropies of order $S \sim \sqrt{k}$, where a Bogomol'nyiPrasad-Sommerfield (BPS) matrix string phase emerges and the diagram is truncated at finite entropy. We find agreement with Vafa's argument [57] that the BPS spectrum in the Ramond (R) sector of the D1-D5 system is that of fundamental type IIB strings carrying winding and momentum (sometimes called Dabholkar-Harvey states [58]). Similarly, chasing through the sequence of dualities for the D1-D5 system on $\mathrm{K} 3 \times \mathrm{S}^{1}$, one finds that the BPS spectrum consists of Dabholkar-Harvey states of the heterotic string.

The character of the phase diagram is different at the extreme limits $q \sim 1$ (i.e. $Q_{1} \sim Q_{5}$ ) and $q \sim k$ (i.e. $Q_{5} \ll Q_{1}$ ). The location of the transition curves bounding the NS5WA patch (type IIA NS five-branes with a wave as the lowenergy description) depend on the ratio $x=\ln q / \ln k$. For roughly equal charges $q \sim 1, x \sim 0$, this patch disappears, as do the related $\overline{\mathrm{M} 5 \mathrm{~W}}$ and M5W patches and the NS5FB patch of fundamental strings and type IIB NS5-branes. The D-brane description predominates the phase diagram, except at low energies where there is a large patch describing fundamental strings with winding and momentum. The opposite regime, say fixed $Q_{5}$ and large $Q_{1}$ so that $x \sim 1$, is the regime discussed by [31]; it is also relevant to the "DLCQ" description of the five-brane [33]. Indeed, the high-entropy region $S>k$ is taken over by the NS5FB patch up to the correspondence curve, while in the low-entropy domain $S<k$, the NS5WA patch expands to squeeze out the $\overline{\mathrm{D} 0 \mathrm{D} 4}, \overline{\mathrm{M} 5 \mathrm{~W}}$, and $\overline{\text { F1WB }}$ patches, and the localized phase is covered by the M5W patch-longitudinal M-theory five-branes with a large boost, just what one needs for an infinite momentum frame or DLCQ description. We discuss the DLCQ limit in detail in Sec. II E below. ${ }^{11}$

For simplicity, we have restricted the set of parameters we have considered in the phase diagram to the entropy and the coupling $g_{6}$. It is straightforward to see what will happen as other moduli of the near-horizon geometry are varied. Consider for instance decreasing one of the $\mathrm{T}^{4}$ radii, keeping the total volume fixed. At some point, the appropriate low energy description will require $T$ duality on this circle, shifting from D1-branes dissolved into D5-branes, to D2-branes, ending on D4-branes. One can then chase this duality around the diagram: The NS5FB phase becomes M2-branes ending on M5-branes; the NS5WA, D0D4, and D0D4 phases become D1-branes, ending on D3-branes; and the $\overline{\mathrm{M} 5 \mathrm{~W}}$ and M5W phases become those of fundamental strings ending on D3branes. The near-extremal F1WB phase is unaffected. One can also imagine replacing the $\mathrm{T}^{4}$ by K3. Moving around the K3 moduli space, when a two-cycle becomes small, a D3brane wrapping the vanishing cycle becomes light; one should consider making a duality transformation that turns $Q_{1}$ or $Q_{5}$ into the wrapping number on this cycle.

\footnotetext{
${ }^{11}$ The relation between the Maldacena conjecture and matrix models of M5-branes has also been considered recently in [59].
} 
Thus the D1-D5 system appears to have a remarkably varied life. On the one hand, it can describe low-energy supergravity on a $6 \mathrm{D}$ space, namely $\mathrm{AdS}_{3} \times \mathrm{S}^{3}$; the common coordinate of the branes is the angle coordinate on $\mathrm{AdS}_{3}$. This space parametrizes physics of the Coulomb branch of the gauge theory. On the other hand, the same system describes the "decoupled" dynamics of the five-brane, another $6 \mathrm{D}$ system $^{12}$ - except that the spatial coordinates are now $\mathrm{T}^{4} \times \mathrm{S}^{1}$, with the $\mathrm{T}^{4}$ apparently related to the physics of the Higgs branch of the gauge theory, and the $S^{1}$ the dimension common to the branes. In the Maldacena limit, the theory is a representation of the $(1+1) \mathrm{D}$ superconformal group; in the DLCQ limit, it describes light-cone M5-branes.

The careful reader will have noted that we have refrained from characterizing the nongravitational dual of the D1-D5 geometry as a $(1+1) \mathrm{D}$ field theory. ${ }^{13}$ The standard candidate for this dual is the $(1+1) \mathrm{D}$ conformal field theory (CFT) on $\operatorname{Sym}^{k}\left(\mathrm{~T}^{4}\right)$ (or K3). This CFT is supposed to provide a description of nonperturbative string theory on $\mathrm{AdS}_{3} \times \mathrm{S}^{3} \times \mathrm{T}^{4}$ (or K3). Indeed, it captures the high-entropy thermodynamics [28] as well as the BPS spectrum $[57,29,60]$. However, the near horizon geometry appears to put the CFT at a singular point in its moduli space $[61,62]$; also, there appears to be a mismatch in the level of the $U(1)^{4}$ affine algebra of Noether charges acting on the $\mathrm{T}^{4}$ [63]. A basic problem also arises in the phase diagram of Fig. 4. In the high-entropy phase, $S$ duality connects the D1-D5 patch to the NS5-F1B patch as one moves to stronger coupling. It is straightforward to check that, crossing the boundary $g_{6}$ $\sim q^{-1 / 2}$, the energy scale of a D1-brane wrapping the torus $\mathrm{T}^{4}$ becomes less than that of a fundamental string; the appropriate effective description is the $S$-dual one. In fact there has to be an entire decuplet of strings transforming under the $O(5,5 ; Z) U$-duality group; the proper low-energy description favors one pair of these, electrically and magnetically charged under one of the five six-dimensional $B$-fields [the subgroup of $U$-duality fixing the description is $\left.{ }^{14} O(5,4 ; Z)\right]$. The problem is that the objects carrying these charges, which are the lightest objects in the theory at intermediate coupling, are not apparent in the $\operatorname{Sym}^{k}\left(\mathrm{~T}^{4}\right)$ symmetric orbifold anywhere on its moduli space. Similarly, in the D1-D5 system on K3 there should be a full $O(5,21) 26$-plet of strings; in this case, tensionless strings corresponding to wrapped D3branes arise when a 2-cycle on the $\mathrm{K} 3$ degenerates, and are

\footnotetext{
${ }^{12}$ Seven-dimensional, if we include the circle transverse to the M5-brane.

${ }^{13}$ The following remarks reflect ongoing discussions of the first author with D. Kutasov and F. Larsen. In particular, it was D. Kutasov that raised the question of whether the dual object is a field theory.

${ }^{14}$ There are BPS charges corresponding to these objects wrapping $\mathrm{T}^{5}$, which are central charges in the 10D supersymmetry algebra. Just as in the case of the transverse five-brane in matrix theory [64], these charges decouple from the supersymmetry algebra in the Maldacena limit; nevertheless the objects remain as finite energy excitations carrying conserved charges.
}

essential in order to regularize the singularities in the effective description. The $(1+1) \mathrm{D}$ CFT on (symmetric products of) $\mathrm{K} 3$ is simply singular, and does not contain the objects which are needed. These objects are carried, however, as fluxes on the five-brane one starts with; the energy cost of these excitations simply becomes small at the relevant points in moduli space, suggesting that the $(5+1) \mathrm{D}$ string-theoretic character of the dynamics does not fully decouple in the Maldacena limit. Similarly, one might expect that lower dimensional examples of the Maldacena conjecture (e.g. those involving $\mathrm{AdS}_{2}$ or $\mathrm{AdS}_{3}$ ) are not fully captured by quantum mechanics or more elaborate $(1+1) \mathrm{D}$ field theories. As mentioned above, it is known that the background fields of the near-horizon limit of the D1-D5 system correspond in the symmetric orbifold CFT to turning off the CFT resolution of the $\mathbf{Z}_{2}$ singularities of $\operatorname{Sym}^{k}\left(\mathrm{~T}^{4}\right)$. It may be that branes wrapping these vanishing cycles are again the needed ingredient for a well-defined description at these points of moduli space.

\section{Spectral flow and angular momentum}

The $(1+1) \mathrm{D} \mathcal{N}=(4,4)$ superconformal algebra has two canonical realizations, depending on whether one chooses antiperiodic (NS) or periodic (R) boundary conditions on the fermionic generators. The spacetime geometry in the Maldacena limit of the D1-D5 system is $\operatorname{AdS}_{3} \times S^{3} \times \mathcal{M}_{4} .2$ $+1 \mathrm{D}$ supergravity on asymptotically locally $\mathrm{AdS}_{3}$ spacetimes carries a realization of this superconformal algebra [56,55]; being a subgroup of the (super)diffeomorphism group, the symmetry extends to the full string theory [31]. $\mathrm{AdS}_{3}$ itself is the vacuum state, and resides in the NS sector since the Killing spinors are antiperiodic; hence low-energy supergravity about this vacuum is described by NS sector representation theory. The $\mathrm{R}$ sector is what one naively discovers as the near-horizon limit of D1-D5 bound states on $\mathcal{M}_{4} \times S^{1}$, since the supercharges are periodic on $S^{1}$.

A similar situation occurs, for example, in D3-brane gauge theory. The gauge theory on $S^{3}$ describes supergravity on $\mathrm{AdS}_{5} \times \mathrm{S}^{5}$ in "global coordinates" [65], where time translation is generated by the dilation operator in the conformal group. The gauge theory on $\mathbb{R}^{3}$ (or $\mathrm{T}^{3}$ ) describes supergravity on a slice of $\mathrm{AdS}_{5} \times \mathrm{S}^{5}$ in "Poincare coordinates" (with periodic identifications for $T^{3}$ ), where time translation is generated by a conformal boost operator. The Poincaré slice is obtained as the limiting near-horizon geometry of black D3-branes in the full string theory. There is no map between gauge theory on $\mathrm{S}^{3}$ and gauge theory on $\mathrm{T}^{3}$.

A major difference in the D1-D5 system is that, since the one-dimensional sphere and torus are the same, the NS and R sectors can be related by a continuous twist of boundary conditions known as spectral flow. This operation shifts conformal dimensions $\left(h_{L}, h_{R}\right)$ and $J_{3}$ charges $\left(j_{L}, j_{R}\right)$ by [66]

$$
\begin{aligned}
& h_{L, R}^{(\eta)}=h_{L, R}^{(0)}-2 \eta j_{L, R}^{(0)}+\eta^{2} k \\
& j_{L, R}^{(\eta)}=j_{L, R}^{(0)}-\eta k .
\end{aligned}
$$

Here, $J_{a}$ are the $\mathrm{SU}(2)$ chiral $R$-symmetry currents of the $\mathcal{N}=(4,4)$ algebra, $E=\frac{1}{2}\left(h_{L}+h_{R}\right)$ is the energy, and $P$ 


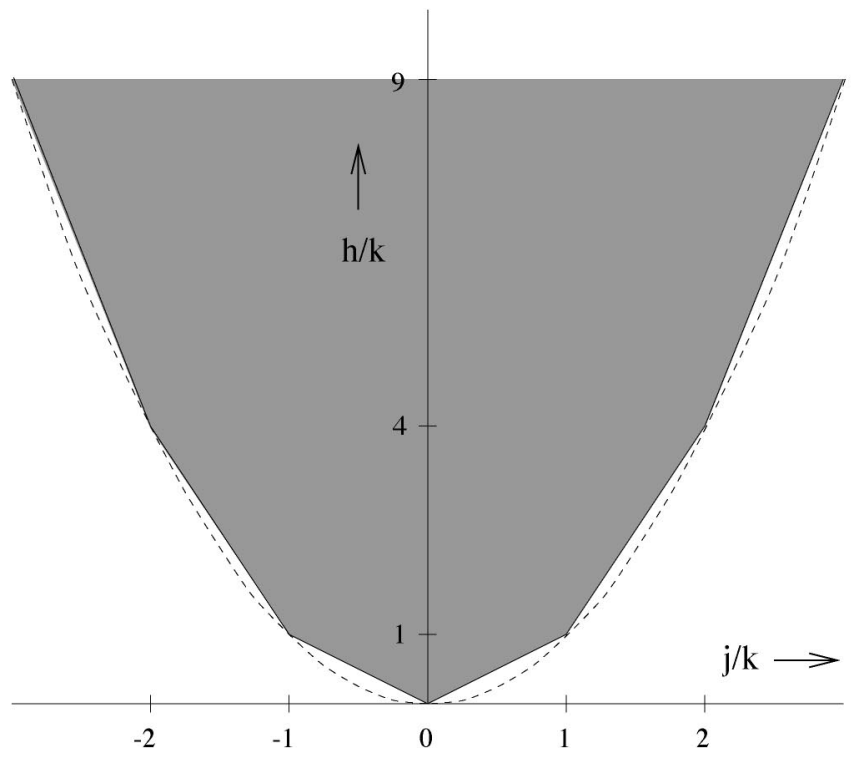

$=\frac{1}{2}\left(h_{L}-h_{R}\right)$ is the momentum along $x_{5}$. We will restrict our attention to the $P=0$ sector. The mode expansions of the supercurrents (which have $j= \pm \frac{1}{2}$ ) are shifted by $\pm \eta$. Thus spectral flow by $\eta=n+\frac{1}{2}, n \in \mathbf{Z}$, relates NS sector states to $\mathrm{R}$ sector states. Moreover, spectral flow by integral amounts $\eta \in \mathbf{Z}$ maps a given sector onto itself; the spectrum maps to itself, but individual states are not preserved. This, combined with the charge conjugation symmetry $j \rightarrow-j$, means that the full spectrum of states in the theory (for both NS and R sectors) with $j_{L}=j_{R}=j$ is determined by, e.g., NS sector states with $0 \leqslant j \leqslant k / 2$. This relation implies a relation between standard conventions in the literature: $h_{(\mathrm{Ram})}=h_{(\mathrm{NS})}$ $-k / 4$, and $j_{(\text {Ram })}=0$ corresponds to $j_{(\mathrm{NS})}=k / 2$.

In fact, there is a simple operation on the full string theory that reduces to spectral flow in the near-horizon limit of the D1-D5 bound state: It is the orbifold described by Rohm [67]. The $S U(2)_{L} \times S U(2)_{R} R$ symmetry of the near-horizon supersymmetry of the D1-D5 system is inherited from the Lorentz group of the asymptotically flat spacetime in which it is embedded in the original string theory. Thus the $R$-symmetry twist is nothing but the imposition of the twisted boundary condition

$$
\Phi\left(x_{5}=R_{5}\right)=\exp \left[i 4 \pi \eta\left(J_{L}^{3}+J_{R}^{3}\right)\right] \Phi\left(x_{5}=0\right) .
$$

In the near-horizon region, the geometry is asymptotically $\mathrm{AdS}_{3} \times \mathrm{S}^{3} \times \mathcal{M}_{4}$, and the spectral flow operation can be understood [68] in the effective Chern-Simons supergravity theory that arises [69]. There, spectral flow is implemented by coupling the $U(1) \times U(1)$ Cartan $R$-symmetry currents to a source; a shift in the energy arises due to the usual relation between regularization (framing) of Wilson line sources and conformal spin in Chern-Simons theory [70]. ${ }^{15}$ It is interesting that, although this twist breaks supersymmetry in the full theory, anti-de Sitter supersymmetry is restored in the near-

\footnotetext{
${ }^{15}$ Thus, very little of the quantum structure of gravity is being
} used here.

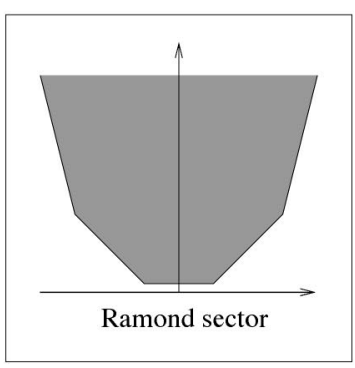

FIG. 5. Allowed region for states belonging to unitary representations of the (NS) superconformal algebra. The dashed curve represents the continuous spectral flow $h_{\eta}=j_{\eta}^{2} / k$ of the point $h=j$ $=0$. Spectral flow slides the boundary polygon along the parabola; a half unit of flow gives the Ramond sector (inset). horizon region; $\eta=\frac{1}{2}$ maps the $\mathrm{R}$ sector of the wrapped brane system to the NS sector, with the R ground state of maximal charge mapping to the NS vacuum.

Unitarity implies that any allowed highest weight representation of the superconformal group must have $h \geqslant|j|[71]$. Spectral flow then implies that allowed states must lie inside the shaded region of the $(h, j)$ plane in Fig. $5 .{ }^{16}$ In particular, spectral flow forces a cutoff on the spectrum of BPS supergravity states (regardless of whether they are single- or multi-particle configurations) at $j=k$; as is easily seen from the figure, states on the line $h=j$ beyond this point lie outside the allowed region (since they would have to flow from states that violate the BPS bound). This feature was termed the "stringy exclusion principle" in [29]; we see that it depends only on some rather mild assumptions about the quantization of Chern-Simons supergravity (i.e. the global structure of the class of geometries under consideration). All such restrictions disappear in the classical $k \rightarrow \infty$ limit.

Spectral flow determines the density of states - at high entropy and far from the boundary of the allowed region-in terms of the Cardy formula $[73,74]$ for zero charge,

$$
S=2 \pi \sqrt{k\left(h_{L}-\frac{1}{4} k\right)-j_{L}^{2}}+2 \pi \sqrt{k\left(h_{R}-\frac{1}{4} k\right)-j_{R}^{2}},
$$

which is precisely the density of states for D1-D5 black holes with angular momentum (remembering the shift in conventions). The expression must be invariant under spectral flow, when the thermal wavelength is much smaller than the size of the system, because the fermion boundary conditions are irrelevant. Near the boundary of the allowed region, the density of states will differ from this expression.

A qualitative sketch of the phase diagram as a function of energy and angular momentum is given in Fig. 6. The loca-

\footnotetext{
${ }^{16}$ These curves are slightly different from the unitarity boundaries of $[71,72]$ since we are only asking that a state be the spectral flow of some state in an allowed representation, rather than that it be an allowed superconformal highest weight.
} 

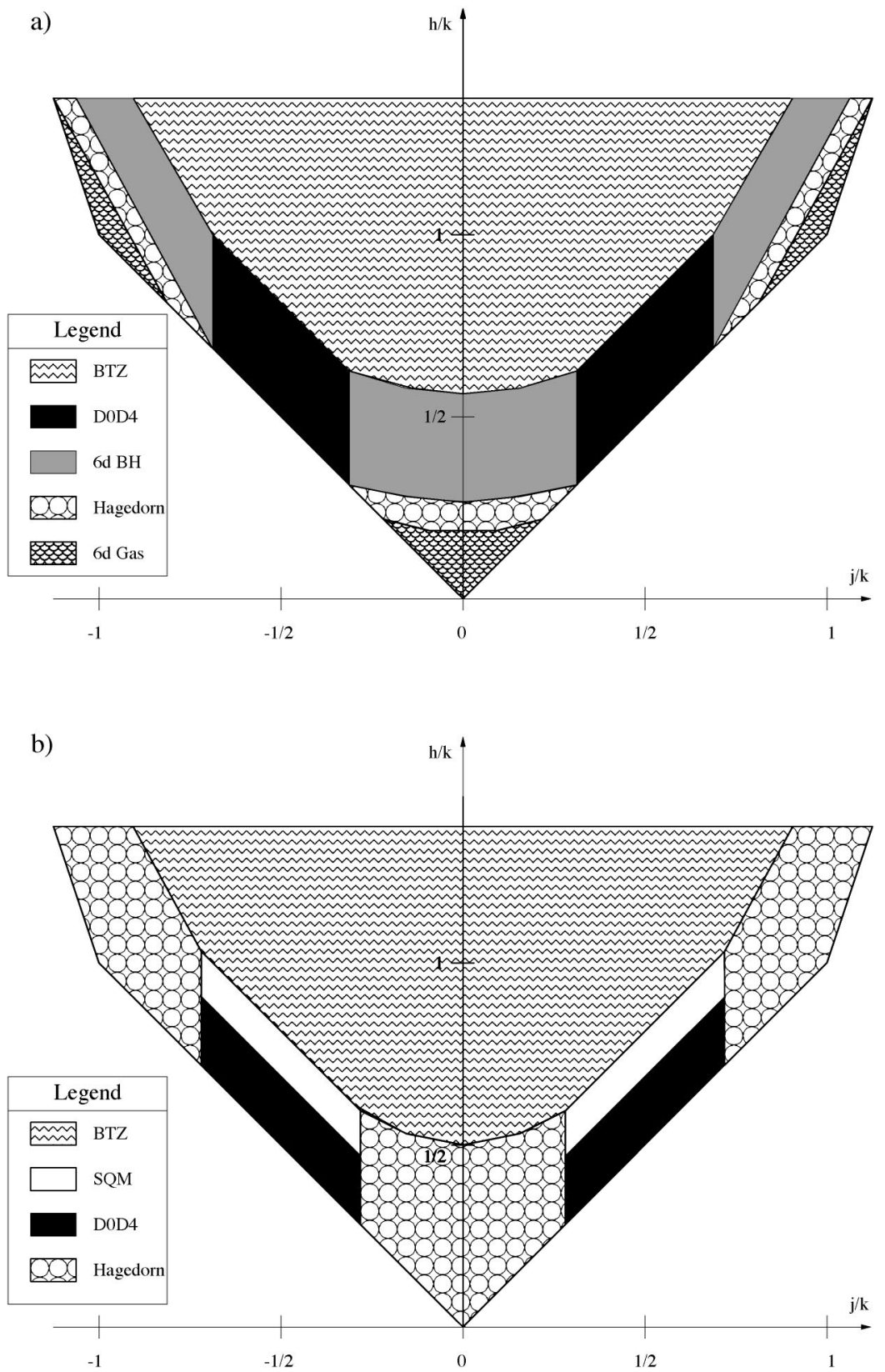

FIG. 6. Qualitative phase diagrams for the D1-D5 system as a function of energy and angular momentum (a) for coupling $g_{\text {eff }} \equiv g_{6}^{2} k>1$, where $g_{6}$ is the six dimensional string coupling; (b) for coupling $g_{\text {eff }}<1$. SQM stands for super quantum mechanics [38], a phase corresponding to energy independent entropy $S \sim k$. tions of the phase boundaries are not precisely determined, ${ }^{17}$ since we only accurately know the phase structure in the vicinity of the $\mathrm{NS}(j=0)$ and $\mathrm{R}(j=k / 2)$ sectors. The $\mathrm{R}$ sector structure is that of Sec. II C, and outlined in the previous section; the NS phase structure was discussed in [75]: There is a "supergravity gas" phase (i.e. the predominant states are dressed Fock space states of low-energy supergravity) about the AdS vacuum; at somewhat higher energy the entropy is dominated by a long string phase; then the string undergoes a correspondence transition to a $(5+1) \mathrm{D}$ Schwarzschild black hole (i.e. localized on $\mathrm{AdS}_{3} \times \mathrm{S}^{3}$ and smeared on $\mathcal{M}_{4}$ ); and finally, at high energy the Banados-

\footnotetext{
${ }^{17}$ Since we are now considering finite $k$, the boundaries between phases are not sharp anyway; they are crossover transitions rather than singularities in derivatives of the free energy.
}

Teitelboim-Zanelli (BTZ) black hole phase with equation of state $(5)$ takes over, as the $(5+1) \mathrm{D}$ black hole delocalizes on $\mathrm{S}^{3}$. As a function of angular momentum, there are then phase boundaries where the NS and R structures abut one another. More details may be found in Sec. II F.

\section{DETAILS FOR THE PHASE DIAGRAMS}

The details of our results can be found in the coming sections. The D4, the D4 on an orbifold, D5, D6 and D1-D5 systems are analyzed in detail in Secs. II A, II B, II C, II D, and II E, respectively. The discussion about spectral flow and angular momentum can be found in Sec. II F.

\section{A. $(2,0)$ theory on $T^{4} \times S^{1}$}

The M5 phase. Our starting point will be D4-branes wrapped on the $\mathrm{T}^{4}$ which is $T$-dual to the matrix theory 
description. This phase consists of six geometrical patches and is described by the equation of state

$$
E \sim \frac{R_{11}}{l_{\mathrm{pl}}^{2}} V^{8 / 5} S^{6 / 5} N^{-3 / 5},
$$

obtained from the geometry of $N$ D4-branes. The geometries are parametrized by the harmonic functions

$$
H=1+\frac{q^{3}}{r^{3}}, \quad h=1-\frac{r_{0}^{3}}{r^{3}},
$$

with

$$
r_{0}^{5} \sim \frac{S^{2}}{N} l_{\mathrm{pl}}^{5} V^{-4}, \quad q^{3} \sim \frac{N}{V^{4}} \frac{l_{\mathrm{pl}}^{5}}{R_{11}^{2}} .
$$

We next describe the six patches of this phase.

The black $D 4$ brane geometry (D4) is given by the metric and dilaton

$$
\begin{aligned}
d s_{10}^{2} & =H^{-1 / 2}\left(-h d t^{2}+d y_{(4)}^{2}\right)+H^{1 / 2}\left(h^{-1} d r^{2}+r^{2} d \Omega_{4}^{2}\right), \\
e^{\phi} & =H^{-1 / 4} .
\end{aligned}
$$

We are using the convention that the asymptotic values of the dilaton are absorbed into the gravitational coupling. The parameters of this geometry are related to the moduli of the light cone $\mathrm{M}$ theory introduced above as follows:

$$
g_{s t r}=\left(\frac{l_{\mathrm{pl}}}{R_{11}}\right)^{1 / 2} V^{-4}, \quad \alpha^{\prime}=\frac{l_{\mathrm{pl}}^{3}}{R_{11}}, \quad y \approx \frac{l_{\mathrm{pl}}^{2} V^{-1}}{R_{11}},
$$

where in the last equation, we use the notation $\approx$ to denote the compactification scale for the four $y$ coordinates, all assumed equal in size. This geometry is subject to the following restrictions:

The Horowitz-Polchinski correspondence principle requires

$$
S>V^{12} N^{-2} .
$$

Otherwise, we connect to a phase described by perturbative $(4+1)$ D SYM. yields

Requiring that the $y$ 's be bigger than the string scale

$$
S>V^{2} N^{4 / 3} .
$$

Otherwise, we $T$ dualize into the geometry of $N$ smeared black D0-branes.

Requiring that small coupling at the horizon yield

$$
S<V^{12} N^{4 / 3} .
$$

Beyond this point, we describe the vacuum via the geometry of black M5-branes.

The $T$-duality transformation yielding the geometry of smeared D0-branes beyond Eq. (12) leads us for $V>1$ onto a phase structure identical to the ones encountered in the three cases studied in [7]. We will therefore be brief in the description of the right half of the phase diagram; a complete discussion can be found in the cited paper. We sketch quickly the scaling of the various transition curves encountered along this chain in the M5 phase.

The smeared black D0 geometry $(\overline{\mathrm{D} 0})$ localizes on the $\mathrm{T}^{4}$ for

$$
S<V^{9 / 2} N^{1 / 2},
$$

into a phase of localized black D0-branes, and gets $\mathrm{M}$ lifted to smeared $M$-theory black waves ( $\overline{\mathrm{W} 11}$ ) at

$$
S \sim N^{4 / 3} V^{-4 / 3} .
$$

At

$$
V \sim 1,
$$

it is seen to be necessary to reduce this latter geometry along one of the cycles of the $\mathrm{T}^{4}$ to the geometry of type IIA waves, then to $T$ dualize on the remaining $\mathrm{T}^{3}$ to a type IIB theory, and finally to $S$ dualize to the geometry of black type IIB waves, to be discussed below. The W11 geometry furthermore collapses at

$$
S \sim N
$$

into the phase described by the geometry of light cone $\mathrm{M}$ theory black holes smeared on the $\mathrm{T}^{4}$.

The black M5 geometry (M5) is obtained from the D4 geometry we started with by lifting it, at strong couplings, to an $\widetilde{M}$ theory. It is described by the metric

$$
d s_{11}^{2}=H^{-1 / 3}\left(d x_{11}^{2}+d y_{4}^{2}-h d t^{2}\right)+H^{2 / 3}\left(h^{-1} d r^{2}+r^{2} d \Omega_{4}^{2}\right),
$$

and the $\widetilde{\mathrm{M}}$ theory is parametrized:

$$
\widetilde{l}_{\mathrm{pl}}^{3}=l_{\mathrm{pl}}\left(\frac{l_{\mathrm{pl}}^{2}}{R_{11}}\right)^{2} V^{-4}, \quad \widetilde{R}_{11}=\frac{l_{\mathrm{pl}}^{2}}{R_{11}} V^{-4}, \quad y_{4} \approx \frac{l_{\mathrm{pl}}^{2}}{R_{11}} V^{-1} .
$$

This geometry is subject to the following restrictions:

Requiring that the curvature at the horizon be greater than the Planck scale $\widetilde{l}_{\mathrm{pl}}$ yields

$$
N>1 \text {; }
$$

i.e., there is no dual geometrical description for $N \sim 1$. Whatever the string theoretical description of a few M5-branes is to be, it will take over the phase description beyond this point.

Requiring that the $\mathrm{T}^{4}$, as measured at the horizon, be bigger than the Planck scale yields the condition

$$
S>V^{-3} N^{4 / 3} .
$$


We must otherwise reduce to the geometry of $\widetilde{\mathrm{D} 4}$ in some type IIA theory residing on $\mathrm{T}^{3} \times \mathrm{S}^{1}$ (where we have isolated an arbitrary one of the four circles to be that of $\mathrm{M}$ reduction to type IIA).

Reducing to $\widetilde{\mathrm{D}} 4$-branes wrapped on $\mathrm{T}^{3} \times \mathrm{S}^{1}$, we find that the size of the $\mathrm{T}^{3}$ as measured at the horizon is smaller than the string scale set by $\tilde{\alpha}^{\prime}$ for entropies satisfying the reverse of Eq. (21). We then $T$ dualize the $\widetilde{\mathrm{D} 4}$-branes to $\widetilde{\mathrm{D} 1}$-branes wrapped on $S^{1}$. We find than the type IIB string coupling measured at the horizon is bigger than one for the reverse of Eq. (21). We then $S$ dualize to the geometry of type IIB black fundamental strings smeared on the $T^{3}(\overline{\mathrm{F} 1})$ :

$$
\begin{aligned}
d s_{10}^{2} & =H^{-1}\left(d x_{11}^{2}-h d t^{2}\right)+d y_{3}^{2}+h^{-1} d r^{2}+r^{2} d \Omega_{4}^{2} \\
e^{\phi} & =H^{-1 / 2} .
\end{aligned}
$$

The type IIB theory is parametrized by

$$
\widetilde{g}_{s}=\frac{l_{\mathrm{pl}} V}{R_{11}}, \quad \widetilde{\alpha}^{\prime}=l_{\mathrm{pl}}^{2} V^{-4}, \quad y_{3} \approx l_{\mathrm{pl}} V^{-2}, \quad \widetilde{R}_{11} \approx \frac{l_{\mathrm{pl}}^{2}}{R_{11}} V^{-4} .
$$

This geometry is the correct dual in this phase provided that:

The curvature at the horizon is smaller than the string scale $\widetilde{\alpha}^{\prime}$ :

$$
S>V^{-3} N^{1 / 2}
$$

Beyond this point, the stringy description is that of a highly excited matrix string, as we will see shortly.

The $\mathrm{T}^{3}$ as measured at the horizon is smaller than the transverse size of the object (set by the angular part of the metric); this yields again Eq. (24). As the box size becomes bigger than the size of the object, the system localizes on the $\mathrm{T}^{3}$. Taking into account the changes to the geometry and thermodynamics as in [7],

$$
\begin{aligned}
d z_{(p)}^{2}+f^{-1} d r^{2}+r^{2} d \Omega_{d}^{2} & \rightarrow f^{-1} d r^{2}+r^{2} d \Omega_{d+p}^{2} \\
r_{0}^{3} & \rightarrow r_{0}^{6} \sim l_{\mathrm{pl}}^{6} S V^{-15 / 2} N^{-3 / 4} \\
q^{3} & \rightarrow q^{6} \sim \frac{l_{\mathrm{pl}}^{8}}{R_{11}^{2}} N V^{-10},
\end{aligned}
$$

we find that the localized fundamental string has its Horowitz-Polchinski point again at Eq. (24). Furthermore, as needed for consistency with this statement, we find that the change in the equation of state for this localized phase does not affect the analysis regarding the matrix string phase we will perform later. Other restrictions on the localized F1 geometry are all seen to be satisfied in the region of the parameter space of interest.

The $\mathrm{T}^{3}$ as measured at the horizon must not be substringy. We find than the size of the torus as measured at the horizon is at the self-dual point.

The size of $x_{11}$ as measured at the horizon is greater than the string scale $\widetilde{\alpha}^{\prime}$ :

$$
S>V^{2} N^{4 / 3}
$$

We otherwise $T$ dualize on $x_{11}$, along the string, and obtain the geometry of smeared type IIB black waves.

Localization on $x_{11}$ is of no concern, since the symmetry structure of the metric does not allow the GregoryLaFlamme localization (25) (i.e. the brane is stretched along this cycle).

The type IIB smeared black wave geometry $(\overline{\mathrm{WB}})$ is the $T$ dual on $x_{11}$ of $\overline{\mathrm{F} 1}$ [Eq. (22)],

$$
\begin{aligned}
d s_{10}^{2}= & (H-1)\left(d x_{11}-d t\right)^{2}+d x_{11}^{2}-d t^{2}+H^{-1}(1-h) d t^{2} \\
& +d y_{3}^{2}+h^{-1} d r^{2}+r^{2} d \Omega_{4}^{2} \\
e^{\phi}= & 1,
\end{aligned}
$$

and the type IIB theory is parametrized by

$\widetilde{g}_{s}=V^{3}, \quad \widetilde{\alpha}^{\prime}=l_{\mathrm{pl}}^{2} V^{-4}, \quad \widetilde{R}_{11} \approx R_{11}, \quad y_{3} \approx l_{\mathrm{pl}} V^{-2}$.

The relevant restrictions are:

Localization on $x_{11}$ occurs at

$$
S \sim N .
$$

The system collapses into a new phase described by the geometry of a boosted type IIB black hole smeared on $\mathrm{T}^{3}$.

The string coupling at the horizon becomes bigger than 1 at

$$
V \sim 1 .
$$

We then are instructed to perform the chain of dualities $S, T_{(3)}, M$, bringing us back to the geometry of light cone M theory black waves $\overline{\mathrm{W} 11}$.

We thus conclude the analysis of the M5 phase. The dual theory can be inferred from the M5 patch; it is the sixdimensional $(2,0)$ theory wrapped on $\mathrm{T}^{4} \times \mathrm{S}^{1}$. Extending the validity of this theory throughout the phase diagram, we conclude that we can interpret it as the phase diagram of the $(2,0)$ theory. We now move onto the other phases of the $(2,0)$ theory; we will be brief in the discussion of the right half of the diagram, since it overlaps in content with the lower dimensional SYM cases [7].

The smeared type IIB black hole (10DBH). This phase is described by the equation of state

$$
E \sim\left(\frac{R_{11}}{N} \frac{1}{l_{\mathrm{pl}}^{2}}\right) V^{8 / 5} S^{8 / 5} .
$$

and consists of the type IIB hole obtained from the type IIB wave geometry $\overline{\mathrm{WB}}$ at $S \sim N$, and the smeared 11D LC hole obtained from the $11 \mathrm{D}$ wave geometry $\overline{\mathrm{W} 11}$. Its correspondence point can be found by minimizing the Gibbs energies between the equation of state (31) and that of the matrix string, which we perform below. The smeared hole geometry localizes on the $\mathrm{T}^{4}$ at 


$$
S \sim V^{9}
$$

where the localized 11D LC black hole emerges.

The black DO phase (D0). This phase consists of the geometries of localized black DO branes (D0) and its M lift light cone $M$ theory waves (W11); the two patches meet at

$$
S \sim N^{8 / 7} .
$$

The equation of state is

$$
E \sim\left(\frac{R_{11}}{N} \frac{1}{l_{\mathrm{pl}}^{2}}\right) S^{14 / 9} N^{2 / 9},
$$

obtained from the D0 geometry. The W11 patch collapses into a light cone $\mathrm{M}$ theory black hole phase at Eq. (17). The black D0-brane patch has its Horowitz-Polchinski correspondence point at $S \sim N^{2}$. This is an interesting transition discussed in greater detail in [7]; on the $S-V$ phase diagram, the $(4+1) \mathrm{D}$ perturbative SYM phase emerges beyond this point.

The $11 \mathrm{D}$ black hole phase (11D BH). The equation of state is given by

$$
E \sim \frac{R_{11}}{l_{\mathrm{pl}}^{2}} N^{-1} S^{16 / 9}
$$

More details about this phase can be found in $[7,76,18]$.

The matrix string phase. The $\overline{\mathrm{F} 1}$ geometry encountered above breaks down via the Horowitz-Polchinski principle of correspondence at Eq. (24). The emerging phase is that of a matrix string. This can be verified as follows: using the string scale given in this geometry (23), we can write down the equation of state of the matrix string phase

$$
E \sim \frac{R_{11}}{l_{\mathrm{pl}}^{2}} N^{-1} S^{2} V^{4}
$$

Matching this energy with that of the M5 phase (6) (or that of the localized F1 geometry) yields Eq. (24). Similarly, we can match the equation of states (36) and that of the type IIB hole (31), yielding the matrix string-boosted type IIB hole transition curve at

$$
S \sim V^{-6}
$$

Perturbative $(4+1) \mathrm{D} S Y M$ phase. The scaling of the equation of state is fixed by dimensional analysis and yields

$$
E \sim\left(\frac{R_{11}}{N} \frac{1}{l_{\mathrm{pl}}^{2}}\right) V N^{1 / 2} S^{5 / 4}
$$

This phase borders that of the D4-branes and D0-branes.

The final phase diagram is that of the $(2,0)$ on $\mathrm{T}^{4} \times \mathrm{S}^{1}$ or, as we see from the LC black hole phase, that of LC M theory on $\mathrm{T}^{4}$.

\section{B. $(2,0)$ theory on $T_{4} / Z_{2} \times S^{1}$}

Inspired by the previous discussion of the phase structure of the $(2,0)$ on $\mathrm{T}^{4} \times \mathrm{S}^{1}$, we further consider the phase structure of this theory on $\mathrm{T}^{4} / Z_{2} \times \mathrm{S}^{1}$. This corresponds to a corner in the moduli space of $\mathrm{K} 3 \times \mathrm{S}^{1}$; particularly, in addition to considering a square $\mathrm{T}^{4}$, we will be ignoring phase dynamics associated with the $16 \times 4$ moduli that blow up the fixed points [77]. Our parameter space is again two dimensional, entropy $S$, and the volume of the $\mathrm{T}^{4}$. There are only two novelties that arise, both leaving the global structure of the phase diagram unchanged, modifying only the interpretation of the various patches of geometry.

The first change arises from the effect of the orbifold on the duality transformations; we will obviously be driven into the other branch of the web of dualities that converge onto $\mathrm{M}$ theory (cf. [78]). We proceed from the $\overline{11 \mathrm{D}}$ phase of the previous discussion, upward and counterclockwise on the phase diagram. We have $\mathrm{M}$ theory on a light-cone circle times $\mathrm{T}^{4} / Z_{2}$. We reduce on $R_{11}$ to D0-branes in type IIA residing on the $\mathrm{T}^{4} / Z_{2}$ at Eq. (15). Under this orbifold, the massless spectrum has positive parity eigenvalue. We $T$ dualize on $\mathrm{T}^{4}$ at Eq. (12), getting to the patch of D4-branes in type IIA wrapped on $\mathrm{T}^{4} / \mathrm{Z}_{2}$. We remind the reader of the transformation

$$
T_{(4)} \beta_{(4)} T_{(4)}^{-1}=\beta_{(4)},
$$

where we have used the properties of the reflection operator on the spinors

$$
\beta_{i}=\Gamma \Gamma^{i}, \quad \beta_{i}^{2}=(-1)^{F_{L}}, \quad\left\{\beta_{i}, \beta_{j}\right\}=0,
$$

with the $T$-duality operation reflecting the left moving spinors only. Here, $(-1)^{F_{L}}$ is the left moving fermion operator. We then $\mathrm{M}$ lift to M5-branes in $\tilde{\mathrm{M}}$ theory on $\mathrm{T}^{4} / Z_{2}$ $\times S^{1}$ at Eq. (13). Next, we have to apply the chain of dualities $M, T_{(3)}, S$ near (21). From the $\mathrm{M}$ reduction we obtain $\widetilde{\mathrm{D}} 4$ branes on $\mathrm{T}^{3} /(-1)^{F_{L}} \Omega$. This is because the $\mathrm{M}$ reduction along an orbifold direction yields the twist eigenvalues, for the massless spectrum,

$$
g_{\mu \nu}+, \quad \phi+, \quad B_{\mu \nu}-, \quad C^{(1)}-, \quad C^{(3)}+,
$$

while the world-sheet parity operator $\Omega$ acts on this spectrum as

$g_{\mu \nu}+, \phi+, B_{\mu \nu}-, C^{(1),(2),(5),(6)}+, C^{(0),(3),(4),(7),(8)}-$,

and the action of $(-1)^{F_{L}}$ yields

$$
\text { NSNS }+, \quad \text { RR }- \text {. }
$$

The $T$ duality on $\mathrm{T}^{3}$ brings us to D1-branes in type IIB theory on $S^{1} \times T^{3} / \Omega$, which is type I theory on $S^{1} \times T^{3}$. This is because

$$
T_{(3)} \beta_{(3)}(-1)^{F_{L} \Omega T_{(3)}^{-1}}=(-1)^{F_{L} \Omega} .
$$

Finally, the $S$ duality culminates in the geometry of $N$ black heterotic strings smeared on the $\mathrm{T}^{3}$. The Horowitz- 
Polchinski correspondence curve (24) patches this phase onto that of the heterotic matrix string phase, whose equation of state obeys the scaling (36). We thus verify the following previous suggestions [44-46,79] from the perspective of Maldacena's conjecture:

Heterotic matrix string theory emerges in the UV of the $(2,0)$ theory.

Heterotic matrix strings can be described via the $O(N)$ SYM of type-I D-strings

The structure of the phase diagram has not changed, but the labelling of some of the phases has. The additional symmetry structure of the orbifold background entered our discussion trivially; the critical behaviors are unaffected.

To complete the discussion, we need to address a second change to the $\mathrm{T}^{4}$ compactification. The localization transitions, say the one occurring at Eq. (14), are of a somewhat different nature than the ones encountered earlier. Localized black geometries on orbifold backgrounds are unstable toward collapse toward the nearest fixed point; by virtue of being above extremality, there are static forces, and by virtue of the symmetry structure of the orbifold, there is no balance of forces as in the toroidal case. It is then most probable that the localized D0-branes sit at the orbifold points, with their black horizons surrounding the singularity. The most natural geometry is the one corresponding to 16 black D0 geometries distributed among the singularities, yielding a nonsingular geometry outside the horizons.

\section{Little strings and five-branes on $\mathbf{T}^{5}$}

In this section, we study the thermodynamics of fivebranes wrapped on a square $\mathrm{T}^{5}$. The notation is as before; we express all equations in terms of the parameters of a LC M theory on $\mathrm{T}^{5}$. The structure of the phase diagram for $V>1$ is similar to the one already encountered. We will therefore not discuss the D0, $\overline{\mathrm{D} 0}$, W11, $\overline{\mathrm{W} 11}, 11 \mathrm{DBH}, \overline{11 \mathrm{DBH}}$, and perturbative $(5+1) \mathrm{D}$ phases except for noting that the only changes to our previous discussion are to Eqs. (15), (31) and (38), which become, respectively,

$$
\begin{aligned}
& S \sim V^{-5 / 2} N^{3 / 2} \\
& E \sim\left(\frac{R_{11}}{N} \frac{1}{l_{\mathrm{pl}}^{2}}\right) V^{5 / 2} S^{3 / 2} \\
& E \sim\left(\frac{R_{11}}{N} \frac{1}{l_{\mathrm{pl}}^{2}}\right) V N^{3 / 5} S^{6 / 5} .
\end{aligned}
$$

We start from the D5 geometry and move counterclockwise on the phase diagram.

The M5 phase ( $\widetilde{\mathrm{M} 5}$ ). This phase consists of seven geometrical patches. For two of these, the $\overline{\mathrm{D} 0}$ and $\overline{\mathrm{W} 11}$, we refer the reader to [7]. The relevant harmonic functions are

$$
H=1+\frac{q^{2}}{r^{2}}, \quad h=1-\frac{r_{0}^{2}}{r^{2}}
$$

with

$$
q^{2} \sim \frac{l_{\mathrm{pl}}^{4}}{R_{11}^{2}} \frac{N}{V^{5}}, \quad r_{0}^{4} \sim \frac{S^{2}}{N} l_{\mathrm{pl}}^{4} V^{-5}
$$

The phase is described by the equation of state ${ }^{18}$

$$
E=\frac{1}{2 \pi} \frac{R_{11}}{l_{\mathrm{pl}}^{2}} S N^{-1 / 2} V^{5 / 2},
$$

characteristic of a string in a Hagedorn phase. Our starting point is the black D5 geometry (D5) given by

$$
\begin{aligned}
d s_{10}^{2} & =H^{-1 / 2}\left(-h d t^{2}+d y_{(5)}^{2}\right)+H^{1 / 2}\left(h^{-1} d r^{2}+r^{2} d \Omega_{3}^{2}\right) \\
e^{\phi} & =H^{-1 / 2}
\end{aligned}
$$

The patch is parametrized by

$$
g_{s t r}=\frac{l_{\mathrm{pl}}}{R_{11}} V^{-5}, \quad \alpha^{\prime}=\frac{l_{\mathrm{pl}}^{3}}{R_{11}}, \quad y_{(5)} \approx \frac{l_{\mathrm{pl}}^{2}}{R_{11}} V^{-1} .
$$

The relevant restrictions are:

The Horowitz-Polchinski correspondence principle be satisfied for

$$
S>V^{15 / 2} N^{-1 / 2} \text {. }
$$

Beyond this point, we sew onto the perturbative (5 $+1)$ D SYM phase whose equation of state is given by Eq. (47).

Requiring that the coupling at the horizon be small yields

$$
S<V^{15 / 2} N^{3 / 2}
$$

We then $S$ dualize to the geometry of black NS5 branes in the type IIB theory.

The condition of large $\mathrm{T}^{5}$ cycles at the horizon requires

$$
S>V^{3 / 2} N^{3 / 2} \text {. }
$$

Otherwise, we $T$ dualize on the $\mathrm{T}^{5}$ and obtain the geometry of smeared D0-branes, $\overline{\mathrm{D} 0}$.

The black NS5 geometry (NS5B) is the $S$ dual of Eq. (51),

$$
\begin{aligned}
d s_{10}^{2} & =-h d t^{2}+d y_{5}^{2}+H\left(h^{-1} d r^{2}+r^{2} d \Omega_{3}^{2}\right) \\
e^{\phi} & =H^{1 / 2}
\end{aligned}
$$

and the new asymptotic moduli are

$$
g_{s t r}=\frac{R_{11}}{l_{\mathrm{pl}}} V^{5}, \quad \alpha^{\prime}=\frac{l_{\mathrm{pl}}^{4}}{R_{11}^{2}} V^{-5}, \quad y_{5} \approx \frac{l_{\mathrm{pl}}^{2}}{R_{11}} V^{-1} .
$$

The relevant restrictions are:

\footnotetext{
${ }^{18}$ Note that we have kept track of the exact numerical coefficient for this equation of state for later use.
} 
Requiring that the cycle size of the five $y$ 's be greater than the string scale yields the condition

$$
V>1
$$

Beyond this point, we need to $T$ dualize on the $\mathrm{T}^{5}$ and we obtain the geometry of black NS5-branes in a type IIA theory.

The correspondence point is at

$$
N \sim 1
$$

We note that the dual theory is the non-local $(1,1)$ theory of type IIB NS5-branes. At low energy, it is described by $(5+1)$ D perturbative SYM.

The geometry of the black NS5 branes in type IIA theory (NS5A) is the $T$ dual of NS5B [Eq. (56)]:

$$
\begin{aligned}
d s_{10}^{2} & =-h d t^{2}+d y_{(5)}^{2}+H\left(h^{-1} d r^{2}+r^{2} d \Omega_{3}^{2}\right) \\
e^{\phi} & =H^{1 / 2} .
\end{aligned}
$$

The parameters of the type IIA theory are

$g_{s t r}=\frac{R_{11}}{l_{\mathrm{pl}}} V^{-5 / 2}, \quad \alpha^{\prime}=\frac{l_{\mathrm{pl}}^{4}}{R_{11}^{2}} V^{-5}, \quad y_{(5)} \approx \frac{l_{\mathrm{pl}}^{2}}{R_{11}} V^{-4}$.

The new restrictions are:

The correspondence point occurs for

$$
N \sim 1
$$

The dual theory is the non-local $(2,0)$ theory of type IIA NS5-branes, related to the $(1,1)$ theory we encountered above via a $T$ duality on the $\mathrm{T}^{5}$.

Requiring small coupling at the horizon yields

$$
S>V^{-15 / 2} N^{3 / 2} \text {. }
$$

Otherwise, we lift to an $\tilde{\mathrm{M}}$ theory and obtain smeared M5-branes.

The smeared black M5 geometry $(\widetilde{\mathrm{M} 5})$ is described by the metric

$$
d s_{11}^{2}=H^{2 / 3}\left(d \widetilde{x}_{11}^{2}+h^{-1} d r^{2}+r^{2} d \Omega_{3}^{2}\right)+H^{-1 / 3}\left(d y_{(5)}^{2}-h d t^{2}\right) .
$$

The parameters of the $\tilde{M}$ theory are

$$
\widetilde{R}_{11}=l_{\mathrm{pl}} V^{-5}, \quad \widetilde{l}_{\mathrm{pl}}^{3}=\frac{l_{\mathrm{pl}}^{5}}{R_{11}^{2}} V^{-10}, \quad y_{(5)} \approx \frac{l_{\mathrm{pl}}^{2}}{R_{11}} V^{-4} .
$$

The new restrictions are:

Requiring that the size of $\tilde{x}_{11}$ as measured at the horizon be smaller than the size of the object gives

$$
S>V^{-15 / 2} N^{1 / 2} \text {. }
$$

Otherwise, we localize in the manner of GregoryLaFlamme on $\tilde{x}_{11}$ to the geometry of localized M5-branes.

The correspondence condition yields

$$
S>V^{-15 / 2} N^{-3 / 2},
$$

which is rendered irrelevant by the previous condition.

Requiring that the cycle size of the $y$ 's at the horizon be bigger than the Planck scale yields

$$
S>V^{3 / 2} N^{3 / 2} \text {. }
$$

Beyond this point, we reduce on one of the cycles of $\mathrm{T}^{5}$ to a type IIA theory. We find that we need to further $T$ dualize on the remaining $\mathrm{T}^{4}$. The resulting geometry of black D0branes is found strongly coupled at the horizon; we therefore lift to another $\hat{\mathrm{M}}$ theory, and we have the geometry of black $\hat{\mathrm{M}}$ waves smeared on the $\mathrm{T}^{4}$.

The geometry of smeared waves in the $\hat{M}$ theory $(\hat{\mathrm{MW}} \overline{\mathrm{W}})$ is given by

$$
\begin{aligned}
d s_{11}^{2}= & (H-1)\left(d \hat{x}_{11}-d t\right)^{2}+d \hat{x}_{11}^{2}-d t^{2}+H^{-1}(1-h) d t^{2} \\
& +d y_{(4)}^{2}+d \tilde{x}_{11}^{2}+h^{-1} d r^{2}+r^{2} d \Omega_{3}^{2} .
\end{aligned}
$$

The parameters of the $\hat{\mathrm{M}}$ theory are

$$
\hat{R}_{11}=R_{11}, \quad \hat{l}_{\mathrm{pl}}=l_{\mathrm{pl}} V^{-2}, \quad y_{(4)} \approx l_{\mathrm{pl}} V^{-2}, \quad \widetilde{R}_{11} \approx l_{\mathrm{pl}} V^{-5}
$$

The relevant restrictions are:

Requiring that the cycle size of $\tilde{x}_{11}$ at the horizon be bigger than the Planck scale $\hat{l}_{\mathrm{pl}}$ yields

$$
V<1 \text {. }
$$

Otherwise, we reduce along $\tilde{x}_{11}$ to a type IIA theory, $T$ dualize on the $\mathrm{T}^{4}$, and $\mathrm{M}$ lift back to the original LC M theory with Planck scale $l_{\mathrm{pl}}$ and five torus moduli $V l_{\mathrm{pl}}$.

The system would localize on $\tilde{x}_{11}$ unless

$$
S>V^{-15 / 2} N^{1 / 2} \text {. }
$$

We then have localized waves in $\hat{\mathrm{M}}$ theory which are still smeared on the remaining $\mathrm{T}^{4}$.

We find that the cycle sizes of the four $y$ 's as measured at the horizon are of order the Planck scale $\hat{l}_{\mathrm{pl}}$.

The system would localize on the $\mathrm{T}^{4}$ unless

$$
S>V^{-3 / 2} N^{1 / 2} \text {. }
$$

This condition is never realized because of the other restrictions.

The system can localize on $\hat{x}_{11}$ unless

$$
S>N
$$

Otherwise, we collapse to the geometry of an 11D black hole in LC $\hat{M}$ theory; this black hole is still smeared on the $\mathrm{T}^{4}$ and on $\tilde{x}_{11}$. 
We thus conclude the discussion of this phase comprised of seven patches. We have two non-local theories sitting on top of the phase, the $(2,0)$ theory and the $(1,1)$ theory, related by a $T$ duality, and bounded by three curves due to finite size effects, and one curve due to the correspondence principle.

The black M5 phase (M5). This phase consists of two patches. The M5 patch (M5) is the localized version of Eq. (64),

$d s_{11}^{2}=H^{2 / 3}\left(h^{-1} d r^{2}+r^{2} d \Omega_{4}^{2}\right)+H^{-1 / 3}\left(d y_{(5)}^{2}-h d t^{2}\right)$,

with the changes

$$
q^{3} \sim \frac{l_{\mathrm{pl}}^{5}}{R_{11}^{2}} N V^{-10}, \quad r_{0}^{5} \sim l_{\mathrm{pl}}^{5} N^{-1} S^{2} V^{-10} .
$$

The equation of state becomes

$$
E \sim \frac{R_{11}}{l_{\mathrm{pl}}^{2}} S^{6 / 5} V^{4} N^{-3 / 5}
$$

In other words, $S \sim N^{1 / 2}\left(y_{(5)} E\right)^{5 / 6}$ in the parameters (65) of this patch; this equation of state is characteristic of a (5 $+1) \mathrm{D}$ gas, as one expects for the theory on the M5-brane at large volume and sufficiently low energy. The new restrictions are:

The correspondence point is now at

$$
N \sim 1 .
$$

Reduction on the $y$ 's along the discussion for the smeared $\widetilde{\text { M5 }}$ branes encountered above occurs at

$$
S \sim N^{4 / 3} .
$$

We then emerge into the phase of MW11 black waves.

The geometry of $11 D$ black waves (M̂W11) is obtained via localization on $\widetilde{x}_{11}$ of the smeared geometry $\hat{M} \bar{W} 11$. The resulting phase is still smeared on the $\mathrm{T}^{4}$. It can however further localize at

$$
S \sim N
$$

along $\hat{x}_{11}$ into a smeared 11D LC black hole $\widehat{11 \mathrm{DB} H}$. The condition of localization on the $\mathrm{T}^{4}$ is, however,

$$
S<N^{1 / 2},
$$

and therefore never arises due to Eq. (80).

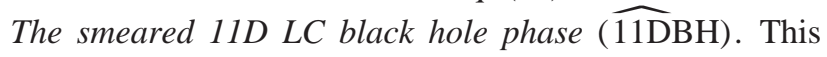
phase is described by the equation of state

$$
E \sim \frac{R_{11}}{l_{\mathrm{pl}}^{2}} N^{-1} V^{4} S^{8 / 5}
$$

It is smeared on the $\mathrm{T}^{4}$ but localized on $\widetilde{x}_{11}$. Minimizing its Gibbs energy as given by Eq. (82) with respect to that of the hole smeared on $\widetilde{x}_{11}$, Eq. (46) yields the transition curve

$$
S \sim V^{-15} .
$$

Localization on the $\mathrm{T}^{4}$ occurs at $S \sim 1$, and therefore is not seen on our phase diagrams. This can be seen by matching Eq. (82) with

$$
E \sim \frac{R_{11}}{l_{\mathrm{pl}}^{2}} N^{-1} V^{4} S^{16 / 9}
$$

i.e. the equation of state of the totally localized hole in the $\hat{\mathrm{M}}$ theory.

This completes the phase diagram obtained from the D5 system. The structure can be verified by using the various equations of state. We conclude by noting that there are several different interpretation of this diagram. It is that of the $(2,0)$ theory; it is that of the $(1,1)$ related to the latter by $T$ duality, but it also encompasses the phase structure of LC M theory on $\mathrm{T}^{5}$. Various previous observations regarding matrix theory on $\mathrm{T}^{5}$ are thus confirmed $[23,22]$ via the Maldacena conjecture.

\section{D6 system}

The Taub-NUT (Newman-Unti-Tamburino) phase. This phase consists of 8 patches. The harmonic functions are

$$
H=1+\frac{q}{r}, \quad h=1-\frac{r_{0}}{r},
$$

with

$$
\begin{aligned}
& r_{0} \sim S^{2 / 3} N^{-1 / 3} V^{-2} l_{\mathrm{pl}} \\
& q \sim \frac{l_{\mathrm{pl}}^{3}}{R_{11}^{2}} N V^{-6} .
\end{aligned}
$$

The equation of state is

$$
E \sim \frac{R_{11}}{l_{\mathrm{pl}}^{2}} S^{2 / 3} N^{-1 / 3} V^{4} .
$$
by

Our starting point is the black D6 geometry (D6), given

$$
\begin{aligned}
d s_{10}^{2} & =H^{-1 / 2}\left(-h d t^{2}+d y_{(6)}^{2}\right)+H^{1 / 2}\left(h^{-1} d r^{2}+r^{2} d \Omega_{2}^{2}\right) \\
e^{\phi} & =H^{-3 / 4} \\
F_{r t y_{1} \cdots y_{6}} & =\partial_{r} H^{-1} .
\end{aligned}
$$

The parameters of this type IIA theory are

$$
\alpha^{\prime}=\frac{l_{\mathrm{pl}}^{3}}{R_{11}}, \quad g_{s}=\left(\frac{l_{\mathrm{pl}}}{R_{11}}\right)^{3 / 2} V^{-6}, \quad y \approx \frac{l_{\mathrm{pl}}^{2}}{R_{11}} V^{-1} .
$$

The various restrictions are:

Weak coupling at the horizon requires

$$
S<N^{2} V^{6} .
$$


Otherwise, we lift to a Taub-NUT geometry in 11 dimensions.

The correspondence point is at

$$
S>V^{6}
$$

Perturbative $6+1 \mathrm{D}$ SYM emerges beyond this point.

$T$ duality on the $y_{(6)}$ must be applied unless

$$
S>N^{2} \text {. }
$$

Otherwise, we have the geometry of smeared $\overline{\mathrm{D} 0}$-branes.

The black Taub-NUT patch in $\bar{M}$ theory ( $\overline{\mathrm{M} T N})$ is given by the geometry

$$
\begin{aligned}
d s_{11}^{2}= & H\left(h^{-1} d r^{2}+r^{2} d \Omega_{2}^{2}\right)+H^{-1}\left(d x_{11}-A d \phi\right)^{2} \\
& -h d t^{2}+d y_{(6)}^{2} .
\end{aligned}
$$

We have introduced a gauge potential $A=(1-\cos \theta) N / 2$ locally $(\theta \neq 0)$ for the magnetically charged 2-form dual to Eq. (88). In the Maldacena limit, this is an 11-dimensional ALE space with $A_{N-1}$ singularity. The parameters of this $\overline{\mathrm{M}}$ theory are

$$
\widetilde{R}_{11}=\frac{l_{\mathrm{pl}}^{3}}{R_{11}^{2}} V^{-6}, \quad \widetilde{l}_{\mathrm{pl}}^{3}=\frac{l_{\mathrm{pl}}^{6}}{R_{11}^{3}} V^{-6}, \quad y_{(6)} \approx \frac{l_{\mathrm{pl}}^{2}}{R_{11}} V^{-1} .
$$

The relevant restrictions are:

The correspondence point

$$
S>N^{-1} V^{6} \text {. }
$$

This is seen to be irrelevant.

The $T^{6}$ must be bigger than the Planck scale:

$$
V>1 \text {. }
$$

Otherwise, we have to reduce along one of the cycles to a type IIA theory, and $T$ dualize along the other five cycles to a type IIB Taub-NUT geometry. We then need to $S$ dualize, and $T$ dualize again on the five torus; finally, we lift to a Taub-NUT geometry in an $\tilde{M}$. Instead of following this path, we will map the $V<1$ region from the $\overline{\mathrm{D} 0}$ geometry.

As mentioned above, we now pick up the trail from it the $\overline{\mathrm{D} 0}$ patch. This patch localizes on the $\mathrm{T}^{6}$ to the D0 geometry for

$$
S>V^{9 / 2} N^{1 / 2},
$$

and lifts to an $M$ theory wave $\overline{\mathrm{W} 11}$ for

$$
S<V^{-6} N^{2}
$$

The latter localizes on the $\mathrm{T}^{6}$ at Eq. (97). For

$$
V<1
$$

we need to reduce the $\overline{\mathrm{W} 11}$ geometry along one of the cycles of the $\mathrm{T}^{6}$, and $T$ dualize on the other five. We find the cou- pling at the horizon is bigger than one, so we $S$ dualize, and find that the $\mathrm{T}^{5}$ is substringy. We $T$ dualize again and find that the resulting type IIA wave geometry is strongly coupled at the horizon. We therefore, and finally, lift to a black wave geometry in an $\tilde{\mathrm{M}}$ theory. The chain of dualities is then $M, T_{5}, S, T_{5}, M$. The new $\tilde{\mathrm{M}}$ wave geometry $(\tilde{\mathrm{M}} \overline{\mathrm{W} 11})$ is parametrized by

$$
z_{(6)} \approx l_{\mathrm{pl}} V^{-5}, \quad \tilde{l}_{\mathrm{pl}}=l_{\mathrm{pl}} V^{-4}, \quad x_{11} \approx R_{11} .
$$

The $\tilde{M}$ circle is one of the $z_{(6)}$, and the wave is along $x_{11}$. This geometry localizes on the $\mathrm{T}^{6}$ for

$$
S<V^{-9 / 2} N^{1 / 2}
$$

The $\mathrm{T}^{6}$ at the horizon is bigger than $\tilde{l}_{\mathrm{pl}}$ for $V<1$, and $x_{11}$ at the horizon is bigger than $\widetilde{l}_{\mathrm{pl}}$ for

$$
S<V^{6} N^{2} \text {. }
$$

Otherwise, we reduce to a type $\widetilde{\text { IIA }}$ theory along $x_{11}$ to the geometry of smeared D0-branes, $\overline{\overline{\mathrm{D} 0}}$. The curvature at the horizon is small with respect to the Planck scale for

$$
S>V^{-3} N^{1 / 2},
$$

which is rendered irrelevant by the other considerations.

The smeared Do geometry $\overline{\overline{\mathrm{D} 0}}$ is parametrized by

$g_{s t r}=\left(\frac{R_{11}}{l_{\mathrm{pl}}}\right)^{3 / 2} V^{6}, \quad \alpha^{\prime}=\frac{l_{\mathrm{pl}}^{3}}{R_{11}} V^{-12}, \quad z_{(6)} \approx l_{\mathrm{pl}} V^{-5}$

A $T$ duality on the $\mathrm{T}^{6}$ takes us to the $\widetilde{\mathrm{D} 6}$ geometry for

$$
S>N^{2}
$$

and localization on the $\mathrm{T}^{6}$ occurs for Eq. (101).

The $\widetilde{D 6}$ geometry is parametrized by

$g_{\text {str }}=\left(\frac{l_{\mathrm{pl}}}{R_{11}}\right)^{3 / 2}, \quad \alpha^{\prime}=\frac{l_{\mathrm{pl}}^{3}}{R_{11}} V^{-12}, \quad z_{(6)} \approx \frac{l_{\mathrm{pl}}^{2}}{R_{11}} V^{-7}$.

This has a correspondence point at

$$
S \sim V^{-6},
$$

and lifts to a Taub-NUT geometry in some $\hat{\mathrm{M}}$ theory for

$$
S>V^{-6} N^{2} \text {. }
$$

The Taub-NUT geometry $\hat{\text { MTN }}$ obtained from the $\widetilde{\mathrm{D} 6}$ patch is parametrized by

$\hat{R}_{11}=\frac{l_{\mathrm{pl}}^{3}}{R_{11}^{2}} V^{-6}, \quad \hat{l}_{\mathrm{pl}}=\frac{l_{\mathrm{pl}}^{2}}{R_{11}} V^{-6}, \quad z_{(6)} \approx \frac{l_{\mathrm{pl}}^{2}}{R_{11}} V^{-7}$.

It patches onto the $\bar{M} T N$ geometry at $V \sim 1$ via a chain of five dualities $M, T_{5}, S, T_{5}, M$ discussed above. 
We note the symmetry of the diagram about $V \sim 1$. The remaining phases were encountered in the previous SYM examples; there is a phase of localized black D0-branes, a LC black hole, a smeared LC black hole, and a $(6+1) \mathrm{D}$ perturbative SYM phase. In the D6 system, each of these phases has a mirror phase about the $V \sim 1$. The structure can be further verified by matching the energies, at fixed entropy, of the various phases. This completes the phase diagram for the D6 system, shown in Fig. 3. We note that:

The gravitational coupling does not vanish in the $\bar{M}$ TN and MTN patches, whereas it does for all the other patches of the diagram.

For $p=5,6$ diagrams involving $(p+1) \mathrm{D}$ SYM, the energy decreases for higher entropies, unlike the $p<5$ cases; i.e., the specific heat is negative.

\section{E. Little strings with winding charge}

We will map here the thermodynamic phase diagram of $Q_{5}$ five-branes and $Q_{1}$ strings. Our starting point is the D1-D5 geometry.

Black five-branes and strings. This phase consists of 12 patches. We start with the D1D5 geometry (D1D5) given by

$$
\begin{aligned}
d s_{10}^{2}= & \left(H_{1} H_{5}\right)^{-1 / 2}\left(-h d t^{2}+d x_{5}^{2}\right)+H_{1}^{1 / 2} H_{5}^{-1 / 2} d x_{(4)}^{2} \\
& +\left(H_{1} H_{5}\right)^{1 / 2}\left(h^{-1} d r^{2}+r^{2} d \Omega_{3}^{2}\right) \\
e^{\phi}= & H_{1}^{1 / 2} H_{5}^{-1 / 2} \\
F_{r t x_{5}}= & \partial_{r}\left(1+\frac{\rho_{1}^{2}}{r^{2}}\right)^{-1}, \quad F_{\theta_{1} \theta_{2} \phi}=2 \rho_{5}^{2}\left(\varepsilon_{3}\right)_{\theta_{1} \theta_{2} \phi} .
\end{aligned}
$$

The harmonic functions are given by

$$
H_{i}=1+\frac{r_{i}^{2}}{r^{2}} \quad i=1,5, \quad h=1-\frac{r_{0}^{2}}{r^{2}}
$$

the charge radii of the branes $\rho_{i}, i=1,5$, are related to the parameters $r_{i}$ by

$$
\begin{gathered}
\rho_{1}^{2}=(2 \pi)^{4} g_{s t r} \alpha^{\prime 3} \frac{(k q)^{1 / 2}}{V_{4}}, \quad \rho_{5}^{2}=g_{s t r} k^{1 / 2} q^{-1 / 2} \alpha^{\prime}, \\
\rho_{i}^{2}=2 r_{i} \sqrt{r_{0}^{2}+r_{i}^{2}} .
\end{gathered}
$$

Here we make a distinction between the antisymmetric tensor field strength's harmonic functions and those of the metric, since we will be interested below in the numerical coefficients of some of the equations of state; the extremal limit corresponds to $r_{0} \rightarrow 0$ with the $\rho_{i}$ held fixed. For scaling purposes, we can write $\rho_{i}=r_{i}$ in the Maldacena limit. We also have traded the two integers $Q_{1}$ and $Q_{5}$ for the new variables $k$ and $q$ :

$$
Q_{1} \equiv \sqrt{k q}, \quad Q_{5} \equiv \sqrt{\frac{k}{q}} .
$$

This type IIB theory is parametrized by $g_{s t r}, \alpha^{\prime}$, and resides on $\mathrm{T}^{4} \times \mathrm{S}^{1}$; the $\mathrm{T}^{4}$ is square with volume $V_{4}$, and we define [29]

$$
\mathrm{V} \equiv \frac{V_{4}}{\alpha^{\prime 2}}, \quad g_{6} \equiv \frac{g_{s}}{\mathrm{v}^{1 / 2}}
$$

The $\mathrm{S}^{1}$ is compact with radius $R_{5}$. The Maldacena limit corresponds to

$$
\alpha^{\prime} \rightarrow 0 \text { with } \frac{r}{\alpha^{\prime}}, \frac{R_{5}}{\alpha^{\prime 1 / 2}}, \quad g_{6} \text { and } \mathrm{v} \text { held fixed. }
$$

This reduces the geometry above to $\mathrm{AdS}_{3} \times \mathrm{S}^{3} \times \mathrm{T}^{4}$. The (1 $+1) \mathrm{D}$ boundary theory is conformal with central charge $c$ $=6 k$. The gravitational coupling in our conventions is

$$
G_{10}=(2 \pi)^{7} g_{s t r}^{2} \alpha^{\prime 4} \text {. }
$$

From the area law, we have

$$
S=\frac{(2 \pi)^{4}}{G_{10}} r_{1} r_{5} r_{0} R_{5} V_{4}
$$

or

$$
r_{0} \sim S g_{s t r} \alpha^{\prime} k^{-1 / 2} \mathrm{v}^{-1 / 2} R_{5}^{-1}
$$

The Arnowitt-Deser-Misner (ADM) mass is

$$
M=\frac{(2 \pi)^{3}}{2} \frac{R_{5} V_{4}}{G_{10}}\left[3 r_{0}^{2}+2\left(r_{1}^{2}+r_{5}^{2}\right)\right],
$$

yielding the equation of state

$$
E=\frac{S^{2}}{8 \pi^{2} k R_{5}}
$$

characteristic of a $(1+1) \mathrm{D}$ conformal field theory [28].

The various restrictions on the D1-D5 geometry are:

The Horowitz-Polchinski correspondence principle dictates

$$
g_{6}>k^{-1 / 2}
$$

Beyond this point, the $(1+1) \mathrm{D}$ conformal theory takes over. Its equation of state is fixed by conformal symmetry; using Cardy's formula $[73,74]$ and the central charge $6 k$, we find precisely Eq. (120), as expected.

Requiring that the coupling at the horizon be small yields

$$
g_{6}<q^{-1 / 2}
$$

Otherwise, we $S$ dualize to the geometry of NS5-branes and fundamental strings.

Requiring the $\mathrm{T}^{4}$ as measured at the horizon be big with respect to the string scale gives

$$
q>1 \text {. }
$$


Otherwise, we apply a $T$ duality on the $\mathrm{T}^{4}$, and exchange the roles of $Q_{1}$ and $Q_{5}$. Without loss of generality, we restrict our attention to $q>1$ only. We also note that $q<k$; the upper bound corresponds to $Q_{5}=1$. We therefore have

$$
1<q<k .
$$

Requiring that the size of $x_{5}$ as measured at the horizon be bigger than the string scale gives

$$
S>g_{6}^{-1 / 2} k^{3 / 4} .
$$

Otherwise, we $T$ dualize to the geometry of smeared D0-D4 branes.

The smeared DOD4 geometry ( $\overline{\mathrm{D} 0 \mathrm{D} 4}$ ) is given by

$$
\begin{aligned}
d s_{10}^{2}= & -\left(H_{1} H_{5}\right)^{-1 / 2} f d t^{2}+H_{1}^{1 / 2} H_{5}^{-1 / 2} d x_{(4)}^{2} \\
& +\left(H_{1} H_{5}\right)^{1 / 2}\left(d x_{5}^{2}+f^{-1} d r^{2}+r^{2} d \Omega_{3}^{2}\right) \\
e^{\phi}= & H_{1}^{3 / 4} H_{5}^{-1 / 4} .
\end{aligned}
$$

The parameters of this type IIA theory become

$$
\begin{gathered}
\widetilde{g}_{s}=g_{s t r} \alpha^{\prime 1 / 2} R_{5}^{-1}, \quad \widetilde{\alpha}^{\prime}=\alpha^{\prime}, \\
x_{(4)} \approx \alpha^{\prime 1 / 2} \mathrm{~V}^{1 / 4}, \quad x_{5} \approx \alpha^{\prime} R_{5}^{-1} .
\end{gathered}
$$

The restrictions are:

Small curvature at the horizon yields the condition

$$
g_{6}>k^{-1 / 2} .
$$

This will be rendered irrelevant by the subsequent conditions.

Small coupling at the horizon requires

$$
S>g_{6}^{1 / 2} k^{3 / 4} q^{1 / 2} .
$$

Otherwise, we lift to the geometry of smeared boosted M5-branes.

Requiring that the size of $x_{5}$ as measured at the horizon be smaller than the transverse size of the object yields

$$
S>g_{6}^{-1} k^{1 / 2} .
$$

Beyond this point, the system localizes in the manner of Gregory and LaFlamme along $x_{5}$, and we have the geometry of localized D0-D4-branes.

Finally, a large $\mathrm{T}^{4}$ is associated with the condition (123).

The smeared boosted M5 geometry ( $\overline{\mathrm{M} 5 \mathrm{~W}}$ ) is the M lift of the $\overline{\mathrm{D} 0-\mathrm{D} 4}$ geometry [Eq. (126)] at Eq. (129):

$$
\begin{aligned}
d s_{11}^{2}= & H_{1}^{-1} H_{5}^{-1 / 3}\left(-f d t^{2}+H_{1} d x_{(4)}^{2}\right) \\
& +H_{5}^{2 / 3}\left(d x_{5}^{2}+f^{-1} d r^{2}+r^{2} d \Omega_{3}^{2}\right) \\
& +H_{1} H_{5}^{-1 / 3}\left[d x_{11}-\left(H_{1}^{-1}-1\right) d t\right]^{2} .
\end{aligned}
$$

The parameters of this $\mathrm{M}$ theory are

$$
\begin{gathered}
R_{11}=g_{s t r} \alpha^{\prime} R_{5}^{-1}, \quad l_{\mathrm{pl}}^{3}=g_{s t r} \alpha^{\prime 2} R_{5}^{-1}, \\
x_{(4)} \approx \alpha^{\prime 1 / 2} \mathrm{v}^{1 / 4}, \quad x_{5} \approx \alpha^{\prime} R_{5}^{-1} .
\end{gathered}
$$

The restrictions are:

The correspondence principle requires

$$
S>g_{6}^{-1} q^{1 / 2} .
$$

This condition is rendered irrelevant by the others for $q$ $<k$. At $q \sim k$, it coincides with the localization condition on $x_{5}$ we will find shortly.

Requiring that the size of $x_{5}$ as measured at the horizon be bigger than the Planck scale yields

$$
S<g_{6}^{-1} k^{3 / 4} q^{-1 / 4}
$$

Otherwise, we reduce along $x_{5}$ to a type IIA theory and to the geometry of boosted NS5-branes.

Requiring that the size of the $\mathrm{T}^{4}$ as measured at the horizon be bigger than the Planck length gives

$$
S>g_{6}^{1 / 2} k^{3 / 4} q^{-1 / 4} \text {. }
$$

Otherwise, we reduce to a type IIA theory along one of the cycles of the $\mathrm{T}^{4}$. We find as always that the other three cycles are substringy and $T$ dualize along them. Finally, the resulting boosted D1 geometry is seen to be strongly coupled at the horizon, and we $S$ dualize to the geometry of boosted type IIB fundamental strings smeared on $x_{5}$. (130)

The localization condition on $x_{5}$ is as for the $\overline{\mathrm{D} 0-\mathrm{D} 4}$ phase

The geometry of NS5 branes and fundamental strings (NS5FB) is obtained from the D1-D5 geometry via $S$ duality:

$$
\begin{aligned}
d s_{10}^{2}= & H_{1}^{-1}\left(-f d t^{2}+H_{1} d x_{(4)}^{2}\right)+H_{1}^{-1} d x_{5}^{2} \\
& +H_{5}\left(f^{-1} d r^{2}+r^{2} d \Omega_{3}^{2}\right) \\
e^{\phi}= & H_{1}^{-1 / 2} H_{5}^{1 / 2} .
\end{aligned}
$$

The parameters of the type IIB theory are

$\widetilde{g}_{s}=g_{s t r}^{-1}, \quad \widetilde{\alpha}^{\prime}=g_{s t r} \alpha^{\prime}, \quad x_{(4)} \approx \alpha^{\prime 1 / 2} \mathrm{v}^{1 / 4}, \quad x_{5} \approx R_{5}$.

The restrictions are:

Small curvature at the horizon requires

$$
k>q,
$$

which is trivially satisfied.

Large $x_{5}$ at the horizon requires

$$
S>k^{3 / 4} q^{1 / 4} .
$$

Otherwise, we $T$ dualize along $x_{5}$ and emerge into the geometry of boosted NS5-branes in type IIA theory; the latter was encountered from the $\overline{\mathrm{M} 5 \mathrm{~W}}$ phase via an M reduction along $x_{5}$.

Large $\mathrm{T}^{4}$ at the horizon requires 


$$
g_{6}<1
$$

Otherwise, we $T$ dualize along the $\mathrm{T}^{4}$, yielding to a similar system with altered asymptotic moduli.

The boosted black type IIB string geometry $(\overline{\mathrm{F} 1 \mathrm{WB}})$ is obtained from the $\mathrm{M} 5 \mathrm{~W}$ patch by a chain of three dualities as described after Eq. (135); this gives the metric

$$
\begin{aligned}
d s_{10}^{2}= & -\left(H_{1} H_{5}\right)^{-1} f d t^{2}+H_{1} H_{5}^{-1} d \hat{x}_{11}^{2}+d x_{(3)}^{2} \\
& +f^{-1} d r^{2}+r^{2} d \Omega_{3}^{2}+d x_{5}^{2} \\
e^{\phi}= & H_{5}^{-1 / 2} .
\end{aligned}
$$

The parameters of the type IIB theory are

$\hat{g}_{s t r}=g_{s t r}^{-1} \alpha^{\prime-1 / 2} \mathrm{v}^{3 / 4} R_{5}, \quad \hat{\alpha}^{\prime}=g_{s t r}^{2} \alpha^{\prime 2} \mathrm{v}^{-1} R_{5}^{-2}$

$x_{(3)} \approx g_{s t r} \alpha^{\prime} \mathrm{v}^{-1 / 2} R_{5}^{-1}, \quad x_{5} \approx \alpha^{\prime} R_{5}^{-1}, \quad \hat{x}_{11} \approx g_{s t r} \alpha^{\prime} R_{5}^{-1}$.

The restrictions are:

Small curvature at the horizon requires

$$
S>k^{1 / 2} \text {. }
$$

This will be rendered irrelevant by other restrictions.

Large $x_{5}$ as measured at the horizon requires

$$
g_{6}<1
$$

Otherwise, we $T$ dualize along $x_{5}$, and obtain a similar geometry.

Localization on $x_{5}$ occurs unless condition (130) is satisfied.

Localization on $x_{(3)}$ occurs unless condition (143) is satisfied. This is irrelevant in view of Eq. (130).

Requiring that $\hat{x}_{11}$ as measured at the horizon be bigger than the string scale yields Eq. (123). (135)

And, finally, we note that the geometry is at the self-dual point for the three cycles $x_{(3)}$.

The geometry of boosted NS5 branes of the type IIA theory (NS5WA) is obtained from the NS5FB patch via $T$ duality or the $\overline{\mathrm{M} 5 \mathrm{~W}}$ via $\mathrm{M}$ reduction. The only relevant restriction is that of large $\mathrm{T}^{4}$ at the horizon. This occurs for

$$
g_{6}<1
$$

Otherwise, we have the $T$ dual, and identical, geometry with different asymptotic moduli.

We have completed the boosted M5 phase up to the condition (145). We note that all duality transformations along $g_{6} \sim 1$ leave the geometries unchanged, and change the asymptotic moduli. It is easy then to check that venturing into domains with $g_{6}>1$ yields a mirrored picture of the phase diagram about $g_{6} \sim 1$. Our six patches have six other mirror geometries across the $g_{6} \sim 1$ line. We therefore see a signature of a strong-weak symmetry $g_{6} \rightarrow 1 / g_{6}$ in the dual theory, which is $T$ duality of the little string. As we scan through $1<q<k$, at the lower bound the phase structure is such that, via dualities exchanging $Q_{1}$ and $Q_{5}$, a mirrored phase diagram for $q<1$ emerges; for the upper bound, the geometrical vacua across the diagram break down via the correspondence principle. These comments carry over to the other phases, which we describe next.

The black localized boosted M5 phase (M5W). The localization transition along $x_{5}$ yields the change in the harmonic functions

$$
f \rightarrow 1-\frac{r_{0}^{3}}{r^{3}}, \quad H_{i} \rightarrow 1-\frac{r_{i}^{3}}{r^{3}},
$$

with

$$
\begin{aligned}
& r_{1}^{3} \sim \frac{\alpha^{\prime 2} g_{s t r}}{\mathrm{v} R_{5}} k^{1 / 2} q^{1 / 2} \\
& r_{5}^{3} \sim \frac{\alpha^{\prime 2} g_{s t r}}{R_{5}} k^{1 / 2} q^{-1 / 2} .
\end{aligned}
$$

The expression (118) for the entropy is unaffected by this transition, unlike all other cases encountered here and in [7]. The equation of state of the localized phase becomes

$$
E \sim \frac{g_{6}}{R_{5}}\left(\frac{S}{k^{1 / 2}}\right)^{3} .
$$

There are three patches. The localized boosted fundamental string $(\mathrm{F} 1 \mathrm{WB})$ is obtained from the $\overline{\mathrm{F} 1 \mathrm{WB}}$ patch by localization on $x_{5}$. The relevant restrictions are:

Small curvature at the horizon requires

$$
S>k^{1 / 2} \text {. }
$$

At this point, we emerge in a matrix string phase carrying two charges. More on this later.

Localization on $x_{(3)}$ occurs unless Eq. (149) is satisfied. This is similar to what we saw in the $(4+1)$ D SYM case.

Large $x_{11}$ at the horizon requires Eq. (123).

Small coupling at the horizon necessitates

$$
S<k^{2 / 3} q^{-1 / 6}
$$

Beyond this point, we apply the chain $S, T_{(3)}, M$ to patch onto the localized boosted M5 geometry. The reverse of this chain was described in the smeared case above.

Finally, the geometry is at the self-dual point for the $x_{(3)}$ cycles.

The localized boosted black M5 brane geometry (M5W) has the same parameters as Eq. (132). It is subject to one additional non-trivial condition, that of $\mathrm{M}$ reduction along $x_{11}$ unless

$$
S<k^{2 / 3} q^{1 / 3}
$$


We then emerge into the geometry of localized D0-D4branes.

The localized D0-D4 brane geometry (D0-D4) is subject to the following additional condition; its curvature at the horizon is small when

$$
S<k
$$

Otherwise, the dual geometrical description breaks down. Comparing the equations of state (148) and (120), we find that we do not have a match at $S \sim k$. This is identical to the situation encountered in all the SYM cases at $S \sim N^{2}$. There is a non-trivial transition at this point through a phase with zero specific heat. On the $(1+1) \mathrm{D}$ gas side, $S \sim k$ is where the thermal wavelength becomes the size of the box, $R_{5}$; the dynamics is then frozen into a quantum mechanics.

The BPS matrix string. At $S \sim k^{1 / 2}$, the emerging phase is that of the Ramond ground states of the conformal theory, which are those of a BPS matrix string. The situation can be compared to the matrix string transition of the M5-brane on $\mathrm{T}^{4} \times \mathrm{S}^{1}$ discussed in Sec. II A. There, we found a correspondence curve at $S \sim V^{-3} Q_{5}^{1 / 2}$, beyond which a perturbative string description should be valid. At the transition, the ratio of the cycle sizes at the horizon of the $\mathrm{T}^{4}$ and the $\mathrm{S}^{1}$ was $\left(y_{4} / \widetilde{R}_{11}\right)^{2} \sim V^{6} \sim Q_{5} / S^{2}$; in particular, since $V \ll 1$, the dynamics is effectively one dimensional. In the localized (M5W) phase of the D1-D5 system, we have $\left(y_{4} / R_{11}\right)^{2}$ $\sim H_{1}^{-1}\left(\alpha^{\prime} \vee^{1 / 2} R_{5}^{2} / g_{s}^{2} \alpha^{\prime 2}\right) \sim Q_{1}^{-1}$ at the transition $S \sim k^{1 / 2}$, which is again of order $Q_{5} / S^{2}$. We conclude that the two transitions are the same. In the present case, the emerging phase is BPS; a perturbative string carrying both winding $Q_{5}$ and momentum $Q_{1}$ quanta obeys the Virasoro constraints

$$
\begin{aligned}
E^{2} & =\left(Q_{1} l_{s t r} / R\right)^{2}+\left(Q_{5} R / 2 l_{s t r}\right)^{2}+2 N_{L}+2 N_{R} \\
k & =Q_{1} Q_{5}=N_{L}-N_{R}
\end{aligned}
$$

when e.g. the left oscillator level $N_{L} \ll N_{R}$, there are of order $k^{1 / 2}$ states, and the system becomes BPS saturated at $N_{R}$ $=0$.

Comments on DLCQ of the M5-brane. As mentioned in the introductory summary, the limit $Q_{5}$ fixed, $Q_{1} \gg Q_{5}$, is relevant to the DLCQ description of the M5-brane [33-36]. In terms of the D1-D5 parameters, the DLCQ parameters are

$$
\begin{aligned}
& \frac{l_{\mathrm{pl}}^{2}}{R_{11}}=l_{\text {str }}\left(\frac{R_{5}}{l_{\text {str }} g_{\text {str }}}\right)^{1 / 3} \\
& \frac{x_{(4)}}{l_{\mathrm{pl}}}=\mathrm{v}^{1 / 4}\left(\frac{R_{5}}{l_{\text {str }} g_{\text {str }}}\right)^{1 / 3} \equiv \nu_{4}^{1 / 4} \\
& \frac{x_{5}}{l_{\mathrm{pl}}}=\frac{l_{\text {str }}}{R_{5}}\left(\frac{R_{5}}{l_{\text {str }} g_{s t r}}\right)^{1 / 3} \equiv L .
\end{aligned}
$$

Converting Eq. (120) to DLCQ parameters, we find

$$
S=2 \pi\left(Q_{5} / L\right)^{1 / 2} l_{\mathrm{pl}} M,
$$

which is indeed the equation of state of a Hagedorn string with tension proportional to $L / Q_{5}$, as has been seen previously from several related points of view [80,81]. It is a nontrivial check that this equation of state agrees precisely with Eq. (50) when we use the parameters (65) of the M5 phase. ${ }^{19}$

The same exercise can be repeated for the localized (M5W) phase. The equation of state (148) in DLCQ parameters, again assuming light-cone kinematics $E_{L C} \sim M^{2} / 2 P$, takes the form

$$
S \sim Q_{5}^{1 / 2} Q_{1}^{1 / 6}\left(\nu_{4}^{1 / 4} l_{\mathrm{pl}} M\right)^{2 / 3}
$$

In terms of scaling, this equation of state is the energyentropy relation of a $(2+1) \mathrm{D}$ gas (extensive in the box size $\left.\nu_{4}^{1 / 4}\right)$, although it is difficult to explain the dependence on $Q_{1}$ and $Q_{5}$. A natural candidate for the object being observed here is an excited M2-brane embedded in the M5-brane (which is indeed one of the bound states of M theory). The $Q_{1}$ dependence appears to violate Lorentz invariance; it would be interesting to understand why light-cone kinematics does not work in the low-energy, low-entropy regime; and why the low-entropy phase is not a boosted version of the $(5+1) \mathrm{D}$ gas found for the M5-brane in Eq. (77).

\section{F. Spectral flow and rotating black holes}

We now turn to a discussion of angular momentum in the D1-D5 system. As pointed out in Sec. ID, spectral flow is an adiabatic twisting of boundary conditions in the full string theory before the Maldacena limit; the near-horizon limit maps the twist onto the spectral flow operation in the superconformal algebra. On the geometry side, a point on the unitarity diagram (Fig. 5) in the NS sector, far from the boundary and at high conformal weight, is described by the BTZ black hole geometry (independent of the fermion boundary conditions) $[82,29,30,83]$, in a space which is asymptotically locally $\operatorname{AdS}^{3} \times S^{3} \times \mathcal{M}_{4}$; in the R sector, such a point represents the near horizon geometry of a rotating D1-D5 system [84] (due to the shift in conventions between canonical definitions of NS and R sector quantum numbers). The isometry $S O(4)=S U(2)_{L} \times S U(2)_{R}$ of the transverse $S^{3}$ combines with the $(4,4)$ supersymmetry generators and the $S L(2, R) \times S L(2, R)$ symmetry of $\mathrm{AdS}_{3}$ to yield two copies of the $\mathcal{N}=4$ superconformal algebra; a gauge transformation in $S U(2)$ can be used to shift the boundary conditions on the supercharges, yielding an isomorphism between the NS and R sectors [66]. The charges under the Cartan subgroups of each of the two $S U(2)$ 's parametrize the angular momenta of the rotating D1-D5 or BTZ hole geometries. The subalgebra of concern is then two copies of the $\mathcal{N}=2$ superconformal algebra with two $U(1) R$-symmetry generators $J_{L, R}^{3}=\frac{1}{2} q_{L, R}^{U(1)} \equiv j$ that implement the spectral flow. We restrict our attention to equal left and right $U(1)$ charges.

\footnotetext{
${ }^{19}$ The light-cone scaling was determined in [81]; our contribution is a check that the precise numerical coefficient agrees.
} 
Consider first the NS sector. The qualitative features of the density of states about $j=0$ were discussed in [75]. There are several phases. Consider the regime of sufficiently large effective coupling $g_{\text {eff }}^{2} \equiv g_{6}^{2} k>1$; in the present notation, for $E R_{A d S}=2 h \geq k$ one is in the BTZ black hole phase [75] with ${ }^{20} S \sim(k h)^{1 / 2}$. For $k g_{\text {eff }}^{-3 / 2} \leq h \lesssim k$, there is a phase of $(5$ $+1) \mathrm{D}$ Schwarzschild black holes because the horizon localizes on $S^{3}$; the entropy is of order $S \sim k^{-1 / 3} h^{4 / 3}$. The lower bound is the correspondence point; thus, for $g_{\text {eff }}^{3 / 2} \lesssim h$ $\lesssim k g_{\text {eff }}^{-3 / 2}$, there is a Hagedorn phase, with $S \sim h g_{\text {eff }}^{-1 / 2}$. Finally, for $h \lesssim g_{\text {eff }}^{3 / 2}$, the system is in a supergravity gas phase, with $S \sim h^{5 / 6}$. At weak coupling $g_{\text {eff }}<1$, the $(5+1) \mathrm{D}$ Schwarzschild phase and the supergravity gas phase disappear.

Consider next the R sector (i.e. $j \approx k / 2$ ), and define $h^{\prime}$ $\equiv h-\frac{1}{4} k$ and $j^{\prime}=j-\frac{1}{2} k$ as the energy and angular momentum in R sector conventions. We first focus on the regime $g_{\text {eff }}>1$, i.e. the middle part of the diagram in Fig. 4. For $g_{\text {eff }}^{-2} k \leq h^{\prime}$, we have the black D1-D5 system. For $0<h^{\prime}$ $\lessgtr g_{\text {eff }}^{-2} k$, we have the M5W or D0-D4 phase localized on $x_{5}$. Finally, at $h^{\prime} \sim 0$, the BPS matrix string cuts the diagram at finite and large entropy $S \sim k^{1 / 2}$. For $g_{\text {eff }}<1$, we have an additional phase with entropy $S \sim k$ for $g_{\text {eff }} k \lesssim h^{\prime} \lesssim k$ squeezed between the D1-D5 and D0-D4 phases. In the phase diagram of Fig. 4, this corresponded to the horizontal line segment at $S \sim k$. As argued in [85], we see that the D1-D5 system without angular momentum does not localize on the $S^{3}$ at low energies, whereas the stationary BTZ hole in the NS sector does undergo such a localization [75]. The spectral flow map adiabatically relates the states of these two sectors; the differing phase structures obtained at zero charge in the NS and $\mathrm{R}$ sectors (the latter flowing to e.g. $j=k / 2$ in the NS sector) then implies that the spinning D1-D5 system must undergo a localization transition on the $S^{3}$ at a critical value of the angular momentum. We next analyze the possibility for such a transition.

The equation of state of the rotating D1-D5 phase can be extracted from the corresponding geometry [84], and is given by Eq. (5)

$$
S_{\mathrm{BTZ}}^{2} \sim k h^{\prime}-j^{\prime 2} .
$$

This phase should collapse at a critical value of $j^{\prime}$ to a (5 $+1) D$ black hole localized and spinning on the $S^{3}$. Angular momentum is introduced in this phase by spinning up the black hole along an orbit on the equator of $S^{3}$ with momentum $p \sim j^{\prime} / R_{\text {AdS }}$; kinematic relations and the Schwarzschild equation of state then imply

$$
S_{6 \mathrm{D}} \sim k^{-1 / 3}\left(h^{\prime 2}-j^{\prime 2}\right)^{2 / 3} \rightarrow k^{-1 / 3} h^{\prime 4 / 3},
$$

\footnotetext{
${ }^{20}$ The standard conventions for the BTZ metric, where length and time scales are referred to the AdS curvature radius, differ from those of the D1-D5 geometry encountered in the R sector, where scales are often referred to the scale $R_{5}$. Matching the asymptotics of the metrics yields the relation $E_{N S} R_{A d S} \sim E_{R} R_{5} \sim h$, where $R_{A d S}^{4}$ $\sim G_{6} k$. We write subsequent equations in terms of the invariant conformal weight $h$ to avoid confusion.
}

where, in the last step, we have taken the non-relativistic limit $h^{\prime} \gg j^{\prime}$; we will see that this is justified. In the relativistic limit the hole approaches extremality; one obtains a gravitational wave on $\mathrm{S}^{3}$ with $h^{\prime} \sim j^{\prime}$, thus matching onto the BPS spectrum of supergravity states. This regime occurs near the boundary of the unitarity plot, where the Hagedorn or gas phase takes over the $(5+1)$ D black hole. The localization on the $\mathrm{S}^{3}$ will then occur if $S_{\mathrm{BTZ}}<S_{6 \mathrm{D}}$ at a given energy, i.e.

$$
\frac{h^{\prime}}{k}<\left(\frac{j^{\prime}}{k}\right)^{2}+\left(\frac{h^{\prime}}{k}\right)^{8 / 3}
$$

For $h^{\prime}<k$, i.e., for the horizon size smaller than the size of the $S^{3}$, we can ignore the last term, and we have the condition

$$
h^{\prime}<\frac{j^{\prime 2}}{k} \text {. }
$$

Note that for $j^{\prime}$ near zero, the corresponding localization condition cannot be met [85]. For $j^{\prime} \sim k$, however, this equation can be satisfied: The direct analysis in the NS sector $j^{\prime}=\frac{1}{2} k$ shows that a localized phase exists at large enough $g_{\text {eff }}$. We conclude that the D1-D5 system indeed localizes on the $S^{3}$ at a critical value of the angular momentum. Note that our uncertainty in the location of the transition is due to the fact that it is sensitive to the numerical accuracy of the equations, not just the scalings of the thermodynamic parameters (and therefore lies beyond the scope of our geometrical analysis). Continuing to lower conformal weights in the $\mathrm{R}$ sector, the equation of state of the rotating D0-D4 phase is given by $[86]$

$$
S^{2} \sim k\left(\frac{h^{\prime}}{g_{6}}\right)^{2 / 3}-j^{\prime 2} .
$$

In the NS sector, the rotating BTZ hole localizes on $\mathrm{S}^{3}$ as the energy is lowered; the equation of state is roughly Eq. (158) (without the primes). As extremality is approached, the spinning black hole reaches the correspondence point and becomes a large fundamental string carrying angular momentum

$$
S_{\mathrm{Hag}} \sim\left[g_{\mathrm{eff}}^{-1 / 2} h-j^{2}\right] .
$$

Below this, there should be a transition where a supergravity gas extremizes the free energy. The phase structure about the NS sector $j \sim 0$ should sew onto the phase structure about the $\mathrm{R}$ sector $j \sim k / 2$ in some intermediate regime. Most of the above formulas are not invariant under spectral flow; they are determined in an analysis about zero angular momentum relative to the NS or R sectors, and may be corrected by large gravitational back reaction when $j \sim k$. We leave an analysis of these effects to future work.

For $g_{\text {eff }}<1$, the picture is slightly different (see Fig. 6). There is no $(5+1) \mathrm{D}$ black hole or supergravity gas phase in the NS sector. The phase labeled SQM has an entropy which is energy independent $S \sim k$. As mentioned above, it corre- 
sponds to the horizontal line at $S \sim k$ in Fig. 4. We again defer a detailed analysis to future work.

It is a curious fact that, for finite $k$, the spectral flow relation between the NS and R sectors implies an IR cutoff in the spectrum of particle states in the latter. In the NS sector, the eigenmodes of the free scalar wave equation have a natural gap in the spectrum of order $1 / R_{A d S} \sim\left(g_{6}^{2} k \alpha^{\prime}\right)^{-1 / 2}$; however, in the $\mathrm{R}$ sector, the free spectrum is continuous. Nevertheless, in the full quantum theory, spectral flow from one sector to the other implies that the finite density of states in the NS sector gives a finite R sector density of states; in other words, finite $k$ generates an effective IR cutoff. This cutoff disappears in the classical $k \rightarrow \infty$ limit, as one sees for example in the fact that the number of BPS states in the R sector is $O(\sqrt{k})$. This feature is a property of all Maldacena- inspired definitions of quantum gravity (i.e. using finite $N$ dual nongravitational systems) where the dual theory is in finite volume (e.g. a torus); the finite density of states due to the IR cutoff in the gauge theory imposes an effective cutoff at large radius in the supergravity - even though the classical wave equations in such geometries can have continuous spectra. It would be interesting to understand this phenomenon better (it is not obviously related to the UV-IR correspondence of [51]).

\section{ACKNOWLEDGMENTS}

We wish to thank J. Harvey, D. Kutasov, F. Larsen, and A. Lawrence for helpful discussions. This work was supported by DOE grant DE-FG02-90ER-40560.
[1] G. T. Horowitz and S. F. Ross, Phys. Rev. D 56, 2180 (1997).

[2] J. M. Maldacena, Nucl. Phys. B (Proc. Suppl.) 61A, 111 (1998).

[3] A. W. Peet, Class. Quantum Grav. 15, 3291 (1998).

[4] J. Maldacena, Adv. Theor. Math. Phys. 2, 231 (1998).

[5] E. Witten, Adv. Theor. Math. Phys. 2, 253 (1998).

[6] S. S. Gubser, I. R. Klebanov, and A. M. Polyakov, Phys. Lett. B 428, 105 (1998).

[7] E. Martinec and V. Sahakian, Phys. Rev. D 59, 124005 (1999).

[8] G. T. Horowitz and J. Polchinski, Phys. Rev. D 55, 6189 (1997).

[9] N. Seiberg, Phys. Rev. Lett. 79, 3577 (1997).

[10] A. Sen, Adv. Theor. Math. Phys. 2, 51 (1998).

[11] S. Hyun, Phys. Lett. B 441, 116 (1998).

[12] S. Hyun and Y. Kiem, Phys. Rev. D 59, 026003 (1999).

[13] T. Banks, W. Fischler, S. H. Shenker, and L. Susskind, Phys. Rev. D 55, 5112 (1997).

[14] L. Susskind, "Another conjecture about M(atrix) theory,", hep-th/9704080.

[15] R. Gregory and R. Laflamme, Phys. Rev. Lett. 70, 2837 (1993).

[16] T. Banks, W. Fischler, I. R. Klebanov, and L. Susskind, Phys. Rev. Lett. 80, 226 (1998).

[17] I. R. Klebanov and L. Susskind, Phys. Lett. B 416, 62 (1998).

[18] G. T. Horowitz and E. J. Martinec, Phys. Rev. D 57, 4935 (1998).

[19] T. Banks, W. Fischler, I. R. Klebanov, and L. Susskind, J. High Energy Phys. 01, 008 (1998).

[20] A. W. Peet and J. Polchinski, Phys. Rev. D 59, 065011 (1999).

[21] L. Susskind, "Holography in the flat space limit," hep-th/9901079.

[22] M. Berkooz, M. Rozali, and N. Seiberg, Phys. Lett. B 408, 105 (1997).

[23] N. Seiberg, Phys. Lett. B 408, 98 (1997).

[24] I. Brunner and A. Karch, Phys. Lett. B 416, 67 (1998).

[25] A. Hanany and G. Lifschytz, Nucl. Phys. B519, 195 (1998).

[26] A. Losev, G. Moore, and S. L. Shatashvili, Nucl. Phys. B522, 105 (1998).
[27] S. Kachru, A. Lawrence, and E. Silverstein, Phys. Rev. Lett. 80, 2996 (1998).

[28] A. Strominger and C. Vafa, Phys. Lett. B 379, 99 (1996).

[29] J. Maldacena and A. Strominger, J. High Energy Phys. 12, 005 (1998).

[30] E. J. Martinec, "Matrix models of AdS gravity," hep-th/9804111.

[31] A. Giveon, D. Kutasov, and N. Seiberg, Adv. Theor. Math. Phys. 2, 733 (1998).

[32] R. Dijkgraaf, E. Verlinde, and H. Verlinde, Nucl. Phys. B486, 77 (1997).

[33] O. Aharony, M. Berkooz, S. Kachru, N. Seiberg, and E. Silverstein, Adv. Theor. Math. Phys. 1, 148 (1998).

[34] N. Seiberg and S. Sethi, Adv. Theor. Math. Phys. 1, 259 (1998).

[35] S. Sethi, Nucl. Phys. B523, 158 (1998).

[36] O. Ganor and S. Sethi, J. High Energy Phys. 01, 007 (1998).

[37] C. V. Johnson, Nucl. Phys. B537, 129 (1999).

[38] J. L. F. Barbon, I. I. Kogan, and E. Rabinovici, Nucl. Phys. B544, 104 (1999).

[39] L. Susskind, "Matrix theory black holes and the gross-witten transition,'” hep-th/9805115.

[40] D. J. Gross and E. Witten, Phys. Rev. D 21, 446 (1980).

[41] L. Motl, "Proposals on nonperturbative superstring interactions," hep-th/9701025.

[42] R. Dijkgraaf, E. Verlinde, and H. Verlinde, Nucl. Phys. B500, 43 (1997).

[43] T. Banks and N. Seiberg, Nucl. Phys. B497, 41 (1997).

[44] M. Berkooz and M. Rozali, Nucl. Phys. B516, 229 (1998).

[45] D.-E. Diaconescu and J. Gomis, Phys. Lett. B 433, 35 (1998).

[46] T. Banks and L. Motl, J. High Energy Phys. 12, 004 (1997).

[47] D. A. Lowe, Phys. Lett. B 403, 243 (1997).

[48] P. Horava, Nucl. Phys. B505, 84 (1997).

[49] R. Dijkgraaf, E. Verlinde, and H. Verlinde, Nucl. Phys. B506, 121 (1997).

[50] N. Itzhaki, J. M. Maldacena, J. Sonnenschein, and S. Yankielowicz, Phys. Rev. D 58, 046004 (1998).

[51] L. Susskind and E. Witten, "The holographic bound in anti-de sitter space,', hep-th/9805114. 
[52] A. Sen, Mod. Phys. Lett. A 11, 827 (1996).

[53] A. Strominger, Phys. Lett. B 421, 139 (1998).

[54] A. Strominger, S.-T. Yau, and E. Zaslow, Nucl. Phys. B479, 243 (1996).

[55] A. Strominger, J. High Energy Phys. 02, 009 (1998).

[56] J. D. Brown and M. Henneaux, Commun. Math. Phys. 104, 207 (1986).

[57] C. Vafa, Nucl. Phys. B463, 415 (1996).

[58] A. Dabholkar and J. A. Harvey, Phys. Rev. Lett. 63, 478 (1989).

[59] H. Awata and S. Hirano, " $A d S_{7} / C F T_{6}$ correspondence and matrix models of M5-branes,", hep-th/9812218.

[60] J. de Boer, Nucl. Phys. B548, 139 (1999).

[61] E. Witten, J. High Energy Phys. 07, 003 (1997).

[62] R. Dijkgraaf, Nucl. Phys. B543, 545 (1999).

[63] D. Kutasov, F. Larsen, and R. G. Leigh, Nucl. Phys. B550, 183 (1999).

[64] T. Banks, N. Seiberg, and S. Shenker, Nucl. Phys. B490, 91 (1997).

[65] G. T. Horowitz and H. Ooguri, Phys. Rev. Lett. 80, 4116 (1998).

[66] A. Schwimmer and N. Seiberg, Phys. Lett. B 184, 191 (1987).

[67] R. Rohm, Nucl. Phys. B237, 553 (1984).

[68] L. Cooper, I. I. Kogan, and R. J. Szabo, Nucl. Phys. B498, 492 (1997).

[69] J. M. Izquierdo and P. K. Townsend, Class. Quantum Grav. 12, 895 (1995).
[70] E. Witten, Commun. Math. Phys. 121, 351 (1989).

[71] D. F. W. Boucher and A. Kent, Phys. Lett. B 172, 316 (1986).

[72] A. Kent and H. Riggs, Phys. Lett. B 198, 491 (1987).

[73] J. L. Cardy, Nucl. Phys. B270, 186 (1986).

[74] D. Kutasov and N. Seiberg, Nucl. Phys. B358, 600 (1991).

[75] T. Banks, M. R. Douglas, G. T. Horowitz, and E. Martinec, "AdS dynamics from conformal field theory," hep-th/9808016.

[76] M. Li, E. Martinec, and V. Sahakian, Phys. Rev. D 59, 044035 (1999).

[77] P. S. Aspinwall, “K3 surfaces and string duality,' hep-th/9611137.

[78] J. Polchinski, String Theory (Cambridge University Press, Cambridge, England, 1998), Vol. 2.

[79] L. Motl and L. Susskind, "Finite N heterotic matrix models and discrete light cone quantization," hep-th/9708083.

[80] J. M. Maldacena, Nucl. Phys. B477, 168 (1996).

[81] O. Aharony and T. Banks, J. High Energy Phys. 03, 016 (1999).

[82] M. Banados, C. Teitelboim, and J. Zanelli, Phys. Rev. Lett. 69, 1849 (1992).

[83] E. J. Martinec, “Conformal field theory, geometry, and entropy,"' hep-th/9809021.

[84] M. Cvetic and F. Larsen, Nucl. Phys. B531, 239 (1998).

[85] A. W. Peet and S. F. Ross, J. High Energy Phys. 12, 020 (1998).

[86] M. Cvetic and D. Youm, Nucl. Phys. B477, 449 (1996). 